\title{
Ultrasound assisted thermal inactivation of spores in foods: Pathogenic and spoilage bacteria, molds and yeasts
}

\author{
Evelyn $^{\text {a,*, Filipa V.M. Silva }}{ }^{\text {b,*** }}$ \\ ${ }^{a}$ Department of Chemical Engineering, University of Riau, Pekanbaru, 28293, Indonesia \\ ${ }^{\mathrm{b}}$ LEAF, Instituto Superior de Agronomia, Universidade de Lisboa, Tapada da Ajuda, 1349-017, Lisboa, Portugal
}

\section{A R T I C L E I N F O}

\section{Keywords:}

Sonoprocessing

Thermosonication

Spore-former

Kinetics

Mathematical models

Pasteurization

\begin{abstract}
A B S T R A C T
Background: Pasteurization aims to achieve the reduction of vegetative microorganisms and resistant spores which are of risk to public health. Power ultrasound has been identified as a potential technology for non-thermal food pasteurization. It relies on the propagation of pressure waves (frequency ranging from 20 to $100 \mathrm{kHz}$ ) of high intensity sound/acoustic energy $\left(10-1000 \mathrm{~W} / \mathrm{cm}^{2}\right)$ inside a liquid or semisolid food, causing inactivation of pathogenic and spoilage microorganisms. This technology can also be used in simultaneous combination with temperature $>80{ }^{\circ} \mathrm{C}$ (thermosonication, TS) to inactivate microbial spores.

Scope and approach: The current knowledge on the effect of ultrasound alone (US), TS, and ultrasound followed by a thermal process (US $\rightarrow \mathrm{T}$ ) on different type of spores relevant for low- and high-acidic foods was reviewed, including the kinetic models describing their inactivation in specific foods.

Key findings and conclusions: US at room temperature (without heat) has no effect on microbial spores. In terms of microbial spore inactivation efficiency, TS was the best method, followed by US $\rightarrow$ T (non-simultaneous application) and finally heat treatment without US (T). TS employing temperatures between $90{ }^{\circ} \mathrm{C}$ and $<100{ }^{\circ} \mathrm{C}$ is required for spore inactivation, as at the boiling point of water ultrasound intensity is reduced. There is a need to design efficient ultrasound probes which can withstand higher temperatures or incorporate pressure to improve gas bubble implosion, thus more results can be produced at higher temperatures and with different type of microorganisms. When reporting results from TS experiments, standardized ultrasound processing conditions including the actual power delivered to the food and expressed in acoustic power density (power/volume of liquid food, $\mathrm{W} / \mathrm{mL}$ ) or specific acoustic power (W/g food) should be used to allow the comparison of results from different studies. TS inactivation kinetics of some spores of bacteria and molds exhibited non-linear trends, thus TS should be examined in detail for reliable food processing.
\end{abstract}

1. Power ultrasound as an attractive technology for the pasteurization of foods

\subsection{Ultrasound fundamentals}

Ultrasound (US) technology can be utilized in important food processing operations such as microbial inactivation, enzyme inactivation, drying, extraction, crystallization, filtration, freezing, emulsification, degassing, tenderization of meat, defoaming and oxidation processes (Awad, Moharram, Shaltout, Asker, \& Youssef, 2012; Silva \& Sulaiman, 2017; Sulaiman, Soo, Farid, \& Silva, 2015). Ultrasound is of great interest for food pasteurization, as it requires lower temperatures than thermal pasteurization alone, it has less impact on food quality, such as nutritional and sensorial characteristics (Charoux, O'Donnell, \& Tiwari, 2017; Tiwari \& Mason, 2012). The main goal of ultrasound pasteurization of foods is to obtain 5 log reductions in target microorganisms (FDA, 2004). As any other pasteurization method, including thermal pasteurization, refrigerated distribution and management is required after the pasteurization process (Silva \& Evelyn, 2018, 2020).

High-power ultrasound also known by power ultrasound is characterized by acoustic waves of low frequency $(20-100 \mathrm{kHz})$ with a typical sound intensity of $10-1000 \mathrm{~W} / \mathrm{cm}^{2}$. It is a known technology used for cell disruptive purposes. According to Gao, Lewis, and Hemar (2016), the extent of microbial inactivation in ultrasonic treatments is mainly

\footnotetext{
* Corresponding author.

** Corresponding author.

E-mail addresses: evelyn@unri.ac.id (Evelyn), filipavinagresilva@gmail.com (F.V.M. Silva).
} 
influenced by three factors: cavitation thresholds (intensity, amplitude, frequency, temperature, and external pressure), media (viscosity, volume, $\mathrm{pH}$, and initial microbial number), and type of microorganism (cell wall, size and shape, spores or vegetative cells, and growth phases). The intensity of the ultrasound process is usually expressed as acoustic power density - APD $\left(\mathrm{W} / \mathrm{mL}\right.$ or $\left.\mathrm{W} / \mathrm{cm}^{3}\right)$ in which the actual power $(\mathrm{W})$ is obtained from a calorimetric experiment or calculated from the ultrasound intensity $\left(\mathrm{W} / \mathrm{cm}^{2}\right)$ dissipated from the probe with a known diameter (Tiwari \& Mason, 2012). When information is limited to the maximum power, the APD may be estimated from the sample volume (assuming no heat loss in the system) and should be stated "less than" since the actual power is lower. The decrease in the power is inversely proportional to the equipment efficiency, which is around $50 \%$. Generally, the ultrasound equipment used in research have a fixed frequency (a value between 20 and $40 \mathrm{kHz}$ ) and the intensity/energy/power of the treatment can be varied by changing the amplitude. Some units can be programmed to be switched on/off for short periods (e.g. $3 \mathrm{~s}$ on $/ 3 \mathrm{~s}$ off) to minimize the thermal impact, lengthen the overall life-time, and save $50 \%$ energy compared to continuous treatments (Tiwari, 2015). With respect to temperature control during US processing, most authors circulated cold water through a jacketed vessel containing the sample to maintain a constant temperature during ultrasound processing. An external pressure can be applied to improve microbial inactivation by ultrasound, manosonication process (Gao et al., 2016). Power ultrasound may also be combined with other treatments such as natural antimicrobials and other preservation technologies such as high hydrostatic pressure and pulsed electric fields to enhance the ultrasound lethal effects and extend a food's shelf life.

\subsection{Mechanism of microbial inactivation}

Cavitation is the phenomenon that occurs during an ultrasound process which refers to the creation, growth, and violent collapse of micro gas bubbles, resulting in turbulence and high-shear forces (Chen, 2012; Feng \& Yang, 2011; Piyasena, Mohareb, \& McKellar, 2004). The collapse of gas bubbles also causes rapid temperature changes, in excess of $5000 \mathrm{~K}$, and $>199 \mathrm{MPa}$ pressure at a micro scale. Cavitation can also cause dissociation of water vapor, producing free radicals as $\mathrm{H}^{+}$and $\mathrm{OH}^{-}$which may recombine to form hydrogen peroxide $\left(\mathrm{H}_{2} \mathrm{O}_{2}\right)$ (Riesz \&
Kondo, 1992). These mechanical, thermal, and chemical effects of cavitation have been suggested to induce stress to microbes, cause destruction of microbial cell structure, and inactivate key enzymes (Butz \& Tauscher, 2002; Chemat, Zill-e-Huma, \& Khan, 2011; Dolas, Saravanan, \& Kaur, 2019). The inactivation of enzymes is related to the depolymerization effect (i.e. degradation of the biological polymers), which causes loss of enzyme activity (Dolas et al., 2019). The main mechanisms of microbial inactivation by ultrasound have been described by Dolas et al. (2019) (Fig. 1). Briefly, the shock waves created by collapsing bubbles shear and break the cell wall and membrane structures, leading to the disruption of the microbial cell components by the micromechanical shocks. Also, free radicals form which react and break the microbe DNA. In addition, the hydrogen peroxide $\left(\mathrm{H}_{2} \mathrm{O}_{2}\right)$ formed during the cavitation process is bactericidal and bacteriostatic. Fig. 2 shows physical alterations in the cellular structure of 2 microbial spores by ultrasound, including disturbed outer layer of spore, cell debris, and disturbed exosporium layer by ultrasound (Palanisamy, Seale, Turner, \& Hemar, 2019). With respect to spore inactivation mechanisms by the simultaneous application of ultrasound and heat (thermosonication, TS), the following has been proposed: (i) release of calcium-dipicolinic acid (DPA), fatty acids, acyl glycerol and glycolipids due to great damage to the spore's exosporium on ultrasonication treatment, further causing a loss of spore resistance, and (ii) a subsequent inactivation by heat (Ansari, Ismail, \& Farid, 2017; Lv, Zou, Chantapakul, et al., 2019; Palacios, Burgos, Hoz, Sanz, \& Ordóñez, 1991).

\subsection{Microbial spore-formers as pasteurization targets in foods}

The classical objective of thermal pasteurization to achieve the inactivation of vegetative forms of microorganisms has been evolving with time. The current definition of pasteurization includes non-thermal pasteurization processes such as power ultrasound and is concerned with the reduction of resistant pathogenic microorganisms of public health risk (NACMCF, 2006; Bermúdez-Aguirre, 2017). Therefore, spore-formers have also become targets in the design of new processes, with recommendations of 5-6 log reductions in the spores, in particular for low-acid foods (Silva \& Gibbs, 2009). Several classes of bacteria and fungi (mold and yeast) are able to produce spores. Bacterial spores are
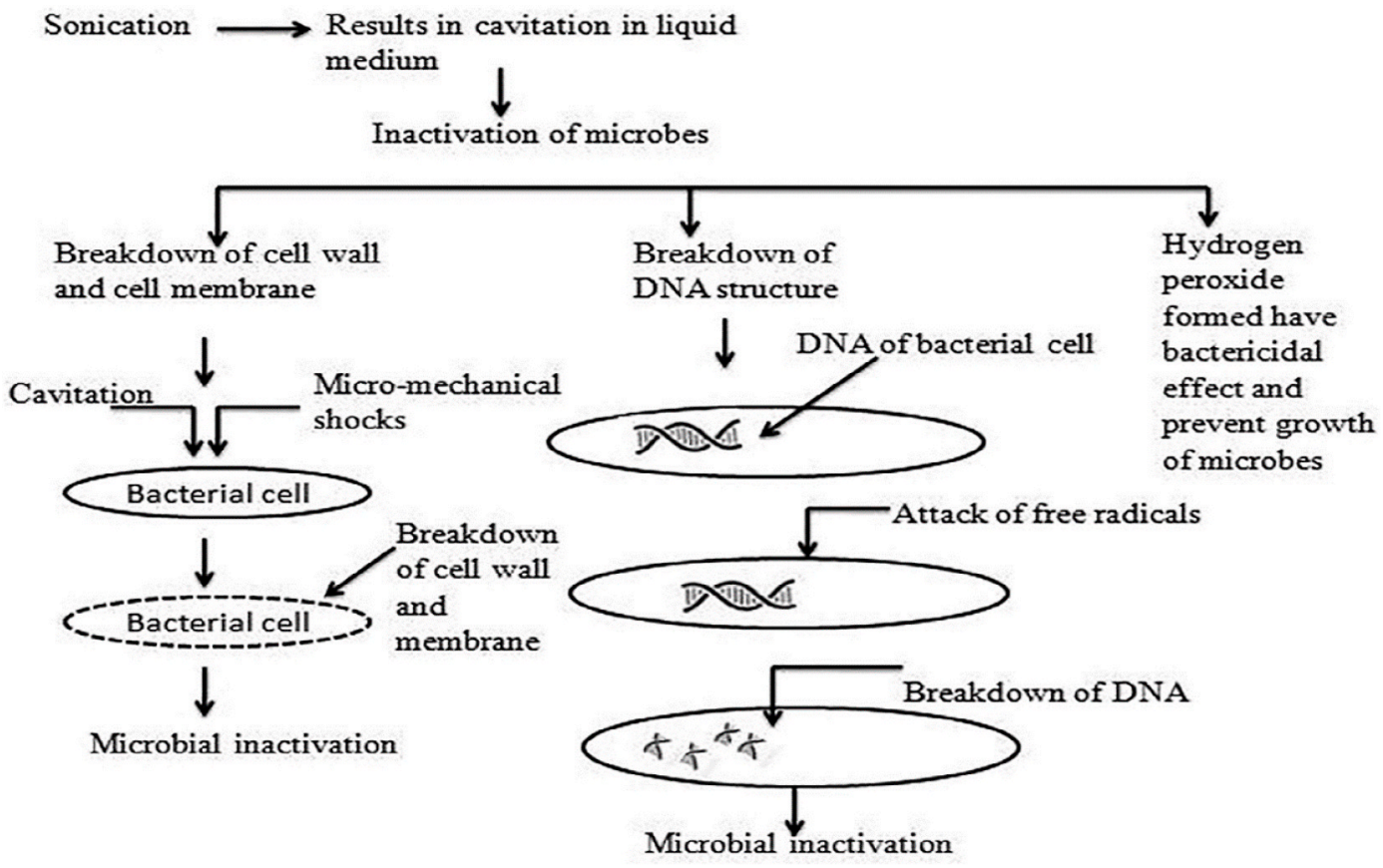

Fig. 1. Mechanism of microbial inactivation by ultrasound (Dolas et al., 2019). Reprinted with permission from Elsevier. 

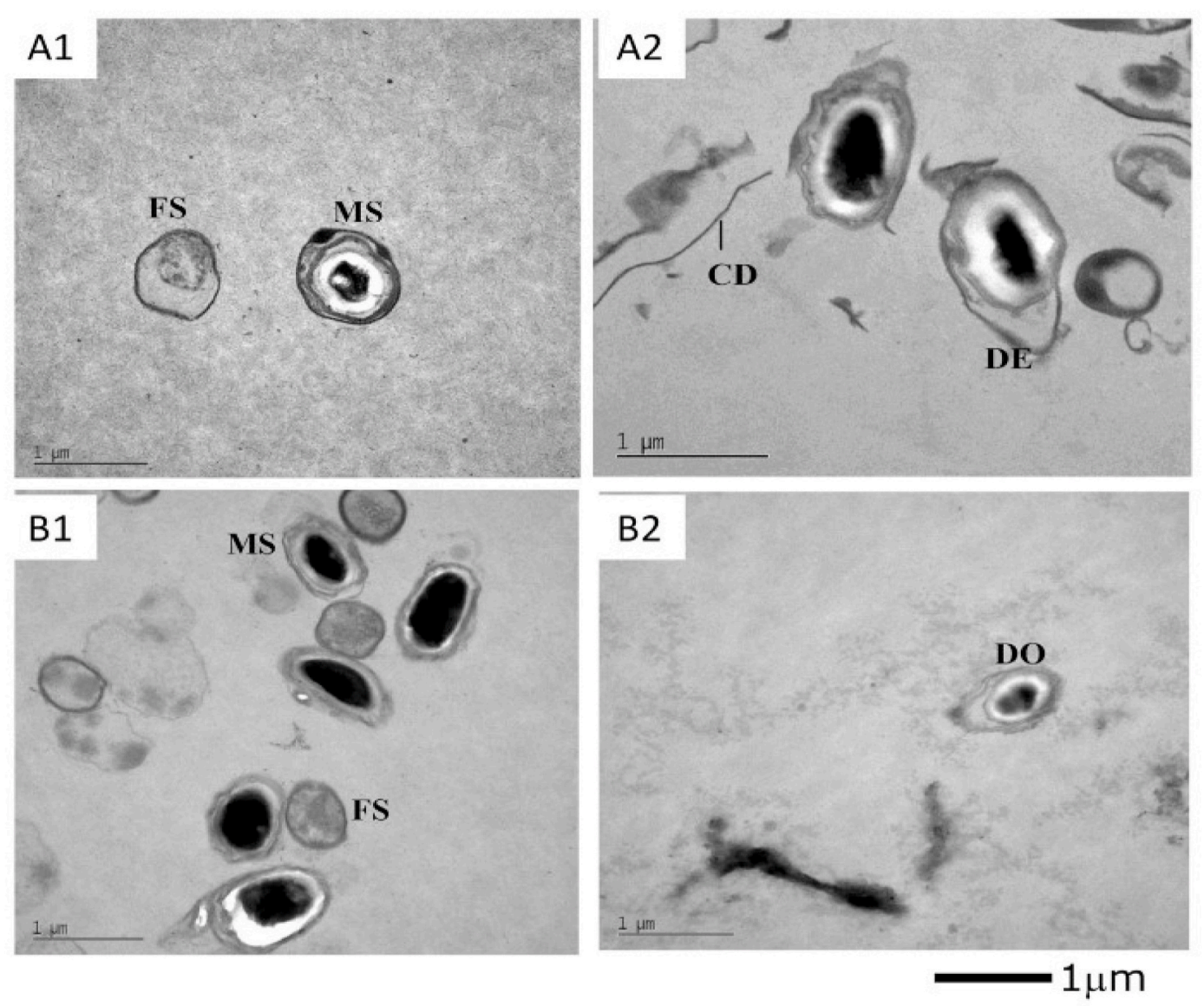

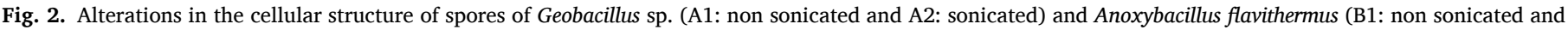

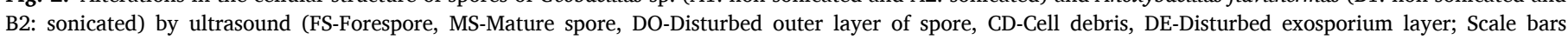
correspond to $0.5 \mu \mathrm{m}$ ) (Palanisamy et al., 2019). Reprinted with permission from Elsevier.

resistant and dormant microbial structures which are formed in response to extreme environmental conditions (e.g. nutrients depletion, shortage of water). The spores help in the survival of these microorganisms for prolonged periods in adverse environments due to its specific morphology, physiology, and chemical composition (Keynan, 1969). Bacterial spores are able to return to vegetative cells through germination induced by sub-lethal heating or nutrients/non-nutrients agents when favorable germination conditions are present. Unlike bacterial spores, fungal spores are part of their reproductive cycles which may be produced either sexually or asexually. Ascospores produced by sexual methods and belonging to ascomycetes sub-kingdom are often more resistant to heat than other forms of fungal spores such as conidiospores and yeast ascopores (Dijksterhuis, 2007; Pitt \& Hocking, 2009). Heat or chemical treatment used alone or in combination can activate fungal spores to germinate into vegetative cells and grow (Sussman \& Halvorson, 1966). The outgrowth of bacterial and fungal spore survivors in foods, can lead to food spoilage, or foodborne illnesses and outbreaks. Therefore, microbial spore-formers have also become targets in food pasteurization and preservation (Silva \& Gibbs, 2001, 2004, 2009, 2010; Evelyn \& Silva, 2018a, 2018b; Silva, Gibbs, Nunez, Almonacid, \& Simpson, 2014; Silva \& Evelyn, 2020). If spores are inactivated, one can assume all vegetative forms are also destroyed. The resistance of spores depends on the type of microbe (bacteria or fungi), the species and the strain.

In this study, the simultaneous application of ultrasound and heat (TS), or the sequential application of ultrasound followed by a thermal process (US $\rightarrow \mathrm{T}$ ) will be reviewed with respect to microbial spore inactivation, as they are considered more effective treatments for the inactivation of resistant spores.

\section{Ultrasound and thermosonication effect on pathogenic and spoilage bacterial spores}

\subsection{Pathogenic bacterial spore-formers}

Clostridium botulinum has been recognized as the most dangerous spore-former bacterium in low-acid foods ( $\mathrm{pH}>4.6)$ (Carlin, Girardin, et al., 2000; Gould, 1999; Lindström, Kiviniemi, \& Korkeala, 2006), producing potent and fatal neuro-intoxication (Brown, 2000). Outbreaks of foodborne botulism in liquid foods such as milk and liquid herbal tea have been reported (Aureli, Fenica, \& Franciosa, 1999; Kim et al., 2019; Therre, 1999; Weingart et al., 2010). Clostridium perfringens and Bacillus cereus are other pathogenic bacteria of concern in low-acid foods. The ability of some strains of $B$. cereus to thrive in temperatures at or below $8{ }^{\circ} \mathrm{C}$ (psychrotrophic) is the reason these strains are found in low-acid chilled foods (Carlin, Guinebretiere, et al., 2000; Dufrenne, Bijwaard, Te Giffel, Beumer, \& Notermans, 1995; Silva et al., 2014). Milk is an example of liquid food which might often be contaminated by $B$. cereus and C. perfringens (Andersson, Ronner, \& Granum, 1995; Chaturvedi \& Shukla, 2015; Feligini et al., 2014). Bacillus licheniformis is another important foodborne disease bacterium. It has been frequently reported as the culprit in milk spoilage (Pavic et al., 2005). Heat and pressure resistance of this bacterium and other Bacillus species were also reported (Janstová \& Lukásová, 2001; Nakayama, Yano, Kobayahi, Ishikawa, \& Sakai, 1996). Other Clostridium and Bacillus bacteria have also become a concern in low-acid foods: Bacillus anthracis, Bacillus pumilus, Bacillus thuringiensis, Clostridium baratii, Clostridium butyricum, and Clostridium difficile (Aureli et al., 1986; Barash, Tang, \& Arnon, 2005; CDC, 2000; Hormazabal \& Granum, 2007; Jackson, Goodbrand, Ahmed, \& Kasatiya, 
Table 1

Inactivation of pathogenic Clostridium and Bacillus spores in low-acid foods and non-food liquids by thermosonication (TS) and ultrasound (US)

\begin{tabular}{|c|c|c|c|c|c|c|c|c|c|}
\hline & & & & Acoustic & \multirow[b]{2}{*}{ Type of } & \multirow[b]{2}{*}{$\mathrm{T}^{\mathrm{b}}$} & \multirow[b]{2}{*}{ Time } & \multirow[b]{2}{*}{ Log } & \\
\hline \multirow[t]{2}{*}{ Species } & \multirow[t]{2}{*}{ Strains } & Medium & Ultrasound & Power Density & & & & & \multirow[t]{2}{*}{ Reference } \\
\hline & & Processed & Conditions & $(\mathrm{W} / \mathrm{mL})^{\mathrm{a}}$ & Treatment & $\left({ }^{\circ} \mathrm{C}\right)$ & $(\min )$ & reduction & \\
\hline \multirow[t]{2}{*}{ Clostridium perfringens } & NZRM 2621 & Beef slurry (pH 6.5) & $24 \mathrm{kHz}$, probe, $210 \mu \mathrm{m}$, & 0.33 & TS & 75 & 20 & 0.9 & Evelyn and Silva \\
\hline & NZRM 898 & & $33 \mathrm{~W}, 100 \mathrm{~g}$ & & & & & 0.9 & $2015 b$ \\
\hline C. perfingens & $\begin{array}{l}\text { Strains tested: } \\
\text { S-80, S-88, 1362, } 3624\end{array}$ & Distilled water & (75 W), probe, $20 \mathrm{~mL}$ & $<3.8$ & US & Ice bath & 12 & 0.0 & $\begin{array}{l}\text { Goodenough and Solberg } \\
1972\end{array}$ \\
\hline Bacillus cereus & ATCC 14579 & Distilled water & $\begin{array}{l}20 \mathrm{kHz}, \text { probe, } \\
(800 \mathrm{~W}), 40 \mathrm{~mL}\end{array}$ & $<20$ & $\begin{array}{l}\text { TS } \\
\text { US }\end{array}$ & $\begin{array}{l}80 \\
\text { Room T }\end{array}$ & $\begin{array}{l}30 \\
30\end{array}$ & $\begin{array}{l}0.5 \\
0.1\end{array}$ & $\begin{array}{l}\text { Lv et al. } \\
2019 \mathrm{a}\end{array}$ \\
\hline B. cereus & ATCC 14579 & Distilled water & $\begin{array}{l}20 \mathrm{kHz} \text {, probe, } \\
1 \mathrm{~min} \text { on } / 2 \mathrm{~min} \text { off, }(200 \mathrm{~W})\end{array}$ & $\mathrm{nr}$ & US & 25 & 7 & 0.0 & $\begin{array}{l}\text { Lv et al. } \\
\text { 2019b }\end{array}$ \\
\hline B. cereus & ATCC 14579 & $\begin{array}{l}\text { Distilled water } \\
(500 \mathrm{~W}), 25 \mathrm{~mL}\end{array}$ & $20 \mathrm{kHz}$, probe, & $<20$ & US & 20 & 30 & 0.9 & $\begin{array}{l}\text { Lv et al. } \\
2020\end{array}$ \\
\hline \multirow[t]{5}{*}{ B. cereus } & $\begin{array}{l}\text { NZRM } 984 \\
\text { (psychrotrophic) }\end{array}$ & $\begin{array}{l}\text { Skim milk (pH 6.5) } \\
\text { Cheese slurry (pH 5.8) }\end{array}$ & $\begin{array}{l}24 \mathrm{kHz} \text {, probe, } \\
0.5 \mathrm{~s} \text { on } / 0.5 \mathrm{~s} \text { off, } 210 \mu \mathrm{m},\end{array}$ & 0.33 & TS & 70 & 1.5 & $<0.5$ & Evelyn and Silva \\
\hline & & Rice porridge (pH 6.7) & $33 \mathrm{~W}, 100 \mathrm{~mL}$ or $100 \mathrm{~g}$ & & & & & 4.1 & \\
\hline & & Beef slurry (pH 6.5) & & & & & & 4.2 & \\
\hline & & Beef slurry (pH 6.5) & & 0.33 & US & 23 & 10 & 0.1 & \\
\hline & & Skim milk (pH 6.5) & & & & & & 0.2 & \\
\hline \multirow[t]{2}{*}{ B. cereus } & $\mathrm{nr}$ & Distilled water & $20 \mathrm{kHz}$, probe, $10 \mathrm{~s}$ on $/ 3 \mathrm{~s}$ off, & $<3$ & TS & 70 & 13.6 & 0.8 & Owusu-Ansah et al. \\
\hline & & Blended pork & $(300 \mathrm{~W}), 100 \mathrm{~mL}$ or $100 \mathrm{~g}$ & & & & & 0.5 & 2020 \\
\hline B. cereus & $\mathrm{nr}$ & $\begin{array}{l}\text { Diluted Ringer } \\
\text { solution }\end{array}$ & $\begin{array}{l}20 \mathrm{kHz} \text {, probe, } \\
(60 \mathrm{~W}), 5 \mathrm{~mL}\end{array}$ & $<12$ & US & $10-12$ & 12 & 0.5 & $\begin{array}{l}\text { Burgos et al. } \\
1972\end{array}$ \\
\hline \multirow[t]{5}{*}{ B. cereus } & Cocktail (ATCC 10876, & Carrot & $40 \mathrm{kHz}$, tank/bath, & Indirect & US & Room T & 5 & $\begin{array}{l}1.3 \\
1.4\end{array}$ & Sagong et al. \\
\hline & (ATCC 13061, W-1) & Lettuce & $40 \mu \mathrm{m}$, glass beaker with & ultrasound & & & & 1.4 & 2013 \\
\hline & & Potato & $0.5 \mathrm{~L}$ sample & & & & & 1.5 & \\
\hline & & Spinach & & & & & & 1.5 & \\
\hline & & Apple/cucumber & & & & & & 1.9 & \\
\hline Bacillus licheniformis & ATCC 6634 & Non-fat milk & $\begin{array}{l}20 \mathrm{kHz}, \text { probe, } 3 \mathrm{~s} \text { on/ } 3 \mathrm{~s} \text { off, } \\
73.6 \mathrm{~W}, 20 \mathrm{~mL}\end{array}$ & 3.7 & US & Ice bath & 10 & 0.2 & $\begin{array}{l}\text { Khanal et al. } \\
2014\end{array}$ \\
\hline B. licheniformis & $\begin{array}{l}\mathrm{nr} \\
\text { solution }\end{array}$ & $\begin{array}{l}\text { Diluted Ringer } \\
(60 \mathrm{~W}), 5 \mathrm{~mL}\end{array}$ & $20 \mathrm{kHz}$, probe, & $<12$ & US & 10-12 & 12 & 0.2 & $\begin{array}{l}\text { Burgos et al. } \\
1972\end{array}$ \\
\hline
\end{tabular}

nr- not reported.

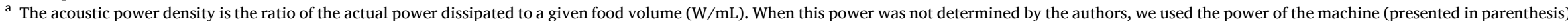
for the calculation and presented the power density with < sign. The paste/semi-solid foods in Evelyn and Silva (2015a, 2015b) were weighed and energy is expressed as a specific acoustic power (W/g).

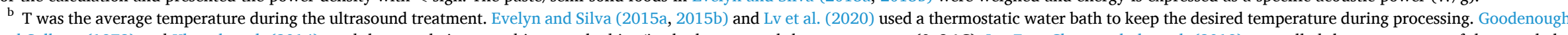

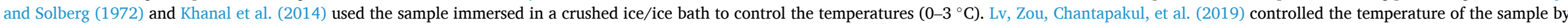

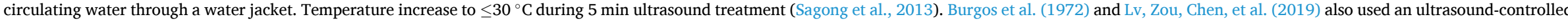
temperature. 
1995; Rupnik \& Songer, 2010; Salkinoja-Salonen et al., 1999).

\subsection{Effect of US and TS on pathogenic Clostridium and Bacillus spores}

Table 1 shows the spore log reductions obtained for pathogenic Clostridium and Bacillus spores in low-acid beverages, other liquids and semisolid foods after ultrasound alone $(0.33$ to $<20 \mathrm{~W} / \mathrm{mL}$ or $\mathrm{W} / \mathrm{g}$ ) or combined with thermal treatments in the range of $70-80{ }^{\circ} \mathrm{C}$. A few authors did not present APD and express the power output in different ways, causing difficult comparison among published literature (Table 1). To overcome this problem, the APD was estimated based on data provided by the authors, assuming 100\% efficient (no heat loss) and a " $<$ " sign was added before APD value estimated.

The analysis of Table 1 shows ultrasound alone (continuous or pulse mode supply of power, and using a probe or bath) was not sufficient to cause significant inactivation of Clostridium and Bacillus spores $(\leq 0.9$ $\log$ ) due to the weak lethal effect of ultrasound alone on the spores ( $\mathrm{Lv}$, Zou, Chen, et al., 2019, 2020, 2019a; Burgos, Ordonez, \& Sala, 1972; Evelyn \& Silva, 2015a; Goodenough \& Solberg, 1972; Khanal, Anand, \& Muthukumarappan, 2014; Malyshev \& Baillie, 2020). Higher log reductions were achieved (up to 1.9) with a cocktail of B. cereus in uncontrolled-room $\mathrm{T}$ and indirect ultrasound treatment, in which increases in the temperature up to $30{ }^{\circ} \mathrm{C}$ during 5 min treatments were reported (Sagong et al., 2013), confirming the important role of heat. Owusu-Ansah et al. (2020) also obtained 0.5-0.8 log for B. cereus in distilled water and blended pork after $<3 \mathrm{~W} / \mathrm{mL}-70{ }^{\circ} \mathrm{C}$ for $13.6 \mathrm{~min}$. Other authors also reported difficulty in inactivating $(0.4 \mathrm{log})$ mesophilic B. cereus ATCC 14579 in distilled water using TS $(<20$ $\mathrm{W} / \mathrm{mL}-80{ }^{\circ} \mathrm{C}$ for $30 \mathrm{~min}$ ) (Lv, Zou, Chantapakul, et al., 2019), similar resistance to US alone ( $\mathrm{Lv}$ et al., 2020). On the other hand, psychrotrophic $B$. cereus NZRM 984 seemed less resistant than the other strains of $B$. cereus, as much higher inactivation (3.2-4.2 log) was achieved in several low-acid foods (cheese slurry, rice porridge, beef slurry) after a TS treatment of $0.33 \mathrm{~W} / \mathrm{g}-70{ }^{\circ} \mathrm{C}$ for $1.5 \mathrm{~min}$ (Evelyn \& Silva, 2015a) as opposed to the higher inactivation of NZRM 984 B. cereus spores could be due to higher heat susceptibility of this psychrotrophic strain, thus demonstrating the strain effect on the inactivation. In addition, the TS inactivation of $B$. cereus spores was also affected by the type of medium, a higher soluble solids content was observed to result in lower spore inactivation (Evelyn \& Silva, 2015a). Thermosonication (TS) of two strains of $C$. perfringens at $0.33 \mathrm{~W} / \mathrm{g}-75^{\circ} \mathrm{C}$ for $20 \mathrm{~min}$ only achieved 0.90 $\log$ in beef slurry (Evelyn \& Silva, 2015b), indicating even higher resistance to the TS treatments. Overall, bacterial spores have high degree of resistance to $75-80{ }^{\circ} \mathrm{C}$ thermosonication. Bacillus spores seemed less resistant to TS treatments and this is in agreement with the previous studies with several Bacillus species (Chandler et al., 2001; Gao et al., 2016; Gao, Lewis, Ashokkumar, \& Hemar, 2014; Ordonez \& Burgos, 1976), especially psychrotrophic group of Bacillus. Experiments testing more US and temperature combinations and $\mathrm{T} \geq 75-80{ }^{\circ} \mathrm{C}$ for inactivating pathogenic Clostridium and Bacillus spores are needed to optimize inactivation and meet the recommended 5D reductions. Previous studies claim the TS intensity decreases as the temperature approaches the water boiling point, due to the high-water vapor tension (Garcia, Burgos, Sanz, \& Ordonez, 1989), which limits the application of TS at high temperatures.

\subsection{Spoilage bacterial spore-formers}

Important spoilage and spore-forming Clostridium in low-acid foods are Clostridium sporogenes, Clostridium esterheticum, Clostridium tyrobutyricum, Clostridium algidicarnis, Clostridium algidixylanolyticum, Clostridium gasigenes, Clostridium beijerinckii, Clostridium laramie, and Clostridium frigidicarnis (Garde, Ávila, Gómez, \& Nuñez, 2013; Ledenbach \& Marshall, 2009). With respect to Bacillus, the following species have been listed as a concern in low-acid foods: B. subtilis, B. atrophaeus (formerly B. subtilis var. niger), thermophilic spore formers (Geobacillus stearothermophilus and Anoxybacillus flavithermus), Bacillus coagulans, Bacillus sphaericus, Bacillus circulans, Bacillus sporothermodurans, Bacillus mycoides, Bacillus megaterium, and Bacillus macerans (Cosentino, Mulargia, Pisano, Tuveri, \& Palmas, 1997; Ledenbach \& Marshall, 2009; Oomes et al., 2007; Palanisamy et al., 2019; Scheldeman, Herman, Foster, \& Heyndrickx, 2006). Both of these Clostridium and Bacillus species have been frequently indicated in the spoilage of meat and dairy products. Although less likely, contamination and growth of spoilage spore-forming Clostridium and Bacillus (C. butyricum, C. tyrobutyricum, Clostridium pasteurianum, B. coagulans, B. licheniformis, B. subtilis, $B$. macerans, $B$. megaterium, and Bacillus polymyxa) have been reported in high-acid foods ( $\mathrm{pH}$ values between 3.7 and 4.5 ) such as mango, mandarin, peach, pear, tomato, and orange fruit pulps and drinks (Azizi \& Ranganna, 1993; De-Jong, 1989; Montville \& Sapers, 1981; Rodriguez, Cousin, \& Nelson, 1993; Sandoval, Barreiro, \& Mendoza, 1992). Growth inhibition of these bacteria in the foods by acidification with citric or ascorbic acids is often employed.

Alicyclobacillus acidoterrestris is another spore-forming bacterium which causes spoilage and subsequent major economic losses in highacid and acidified foods $(\mathrm{pH}<4.6)$ (Cerny, Duong, Hennlich, \& Miller, 2000; Jay, 2000; Silva \& Evelyn, 2018). The optimal growth $\mathrm{pH}$ and temperature for this bacterium is between 3.5 and 4.5 and between 35 and $53{ }^{\circ} \mathrm{C}$, respectively (Deinhard, Blanz, Poralla, \& Altan, 1987; Pinhatti, Variane, Eguchi, \& Manilla, 1997). Examples of foods associated with $A$. acidoterrestris spoilage are shelf-stable aseptically packaged apple juice, carbonated fruit juice drinks, fruit pulps, isotonic water, lemonade, and shelf-stable ice tea containing berry juice (Cerny et al., 2000; Duong \& Jensen, 2000; Pettipher \& Osmundson, 2000; Walls \& Chuyate, 1998).

\subsection{Effect of US and TS on spoilage bacterial spores}

Table 2 shows the inactivation of spoilage bacterial spores in lowand high-acid foods and non-food liquids after ultrasound and TS with temperatures from 60 up to $100^{\circ} \mathrm{C}$ for $0.3-60 \mathrm{~min}$. Similar to pathogenic spores, ultrasound treatments at room $\mathrm{T}$ for up to $30 \mathrm{~min}$ were not able to inactivate several spoilage species of bacterial spores $(\leq 0.3 \log )$ (Broda, 2007; Fan, Ismail, Hou, Muhammad, Zou, et al., 2019; Ferrario, Alzamora, \& Guerrero, 2015; Khanal et al., 2014; Tremarin, Brandão, \& Silva, 2017), although for one study 1.3-1.5 decimal reductions were registered (Palanisamy et al., 2019). Extension of US processing times to up to $60 \mathrm{~min}$ led to a reduction of $0.8 \log$ of $A$. acidoterrestris (Tremarin, Canbaz, Brandão, \& Silva, 2019). TS inactivation of G. stearothermophilus in skim milk containing $31.5 \%$ total solids at $60{ }^{\circ} \mathrm{C}$ for 0.3 min only resulted in $0.3 \mathrm{log}$ (Beatty \& Walsh, 2016). TS-73 ${ }^{\circ} \mathrm{C}-2 \mathrm{~min}$ also only resulted in a $0.1-0.2 \log$ inactivation of B. subtilis, G. stearothermophilus, and A. flavithermus in $2 \%$ fat milk/tryptic soy broth (Deshpande \& Walsh, 2020), whereas TS-78 ${ }^{\circ} \mathrm{C}-30$ min resulted in a 1.0 $\log$ inactivation of $A$. acidoterrestris in orange juice (Evelyn \& Silva, 2016). Fan, Ismail, Hou, Muhammad, Zou, et al. (2019) worked with $B$. subtilis in water and obtained a $1.8 \mathrm{log}$ after TS at $80{ }^{\circ} \mathrm{C}$ for $20 \mathrm{~min}$. Ganesan, Martini, Solorio, and Walsh (2015) reported 2.0 log reduction of B. atrophaeus spores in UHT milk submitted to TS at $84.8^{\circ} \mathrm{C}$ for 5.8 min. Thus, long processing times will be needed to achieve a $5 \mathrm{D}$ reduction in the target microorganism.

Thermosonication at higher temperatures appeared to produce better results. For example, Tremarin et al. (2017) obtained 5.0-5.5 log reduction of (10 days and 6-month old) spores of $A$. acidoterrestris in commercial apple juice $\left(11^{\circ} \mathrm{Brix}\right)$ when using a TS temperature of $95^{\circ} \mathrm{C}$ for $20 \mathrm{~min}$. Garcia et al. (1989) could achieve 5.5-6.3 log reductions of two B. subtilis strains in whole milk after a TS treatment at $100{ }^{\circ} \mathrm{C}$ for 10 min, indicating the important role of temperature in spore inactivation during TS treatments. These authors also observed a large increase in the microbial resistance to TS when using glycerol ( $\sim$ only 1 log reduction), as this compound caused a protective effect in the spores. The increase of TS temperature $\left(>90^{\circ} \mathrm{C}\right)$ resulted in a higher inactivation of bacterial 
Table 2

Inactivation of spoilage bacterial spores in low- and high-acid foods and non-food liquids by thermosonication (TS) and ultrasound (US).

\begin{tabular}{|c|c|c|c|c|c|c|c|c|c|}
\hline \multirow[t]{2}{*}{ Species } & \multirow[t]{2}{*}{ Strains } & \multirow{2}{*}{$\frac{\text { Medium }}{\text { Processed* }^{*}}$} & \multirow{2}{*}{$\frac{\text { Ultrasound }}{\text { Conditions }}$} & \multirow{2}{*}{$\frac{\text { Acoustic Power Density }}{(\mathrm{W} / \mathrm{mL})^{\mathrm{a}}}$} & \multirow{2}{*}{$\frac{\text { Type of }}{\text { Treatment }}$} & \multirow{2}{*}{$\frac{\mathrm{T}^{\mathrm{b}}}{\left({ }^{\circ} \mathrm{C}\right)}$} & \multirow{2}{*}{$\frac{\text { Time }}{(\mathrm{min})}$} & \multirow{2}{*}{$\frac{\log }{\text { Reduction }}$} & \multirow[t]{2}{*}{ Reference } \\
\hline & & & & & & & & & \\
\hline \multicolumn{10}{|c|}{ Low-acid foods and non-food liquids } \\
\hline Clostridium estertheticum & DSM 8809 & Phosphate buffer & $\begin{array}{l}20 \mathrm{kHz}, 1 \mathrm{~min} \text { on } / 5 \mathrm{~min} \text { off, } \\
(90 \mathrm{~W}) \text {, probe }\end{array}$ & $\mathrm{nr}$ & US & Ice bath & 3 & 0.0 & $\begin{array}{l}\text { Broda } \\
2007\end{array}$ \\
\hline Bacillus subtilis & $\begin{array}{l}\text { var niger-40 } \\
\text { ATCC } 6051\end{array}$ & Whole milk & $20 \mathrm{kHz},(150 \mathrm{~W}), 30 \mathrm{~mL}$ & $<5$ & TS & 100 & 10 & $\begin{array}{l}5.5 \\
6.3\end{array}$ & $\begin{array}{l}\text { Garcia et al. } \\
1989\end{array}$ \\
\hline B. subtilis & ATCC 6633 & Water & $\begin{array}{l}20 \mathrm{kHz} \text {, probe, }(600 \mathrm{~W}), \\
30 \mathrm{~mL}\end{array}$ & $\begin{array}{l}<20 \\
<20\end{array}$ & $\begin{array}{l}\text { TS } \\
\text { US }\end{array}$ & $\begin{array}{l}80 \\
23\end{array}$ & $\begin{array}{l}20 \\
20\end{array}$ & $\begin{array}{l}1.8 \\
0.0\end{array}$ & $\begin{array}{l}\text { Fan et al. } \\
2019 \mathrm{a}\end{array}$ \\
\hline B. subtilis & DSM 618 & $\begin{array}{l}\text { Tryptic soy broth } \\
\text { Milk ( } 2.0 \% \text { fat })\end{array}$ & $\begin{array}{l}20 \mathrm{kHz}, \text { probe, } \\
11 \mathrm{~W}, 5 \mathrm{~mL}\end{array}$ & 2.2 & TS & 73 & 2 & $\begin{array}{l}0.1 \\
0.2\end{array}$ & $\begin{array}{l}\text { Deshpande and Walsh } \\
2020\end{array}$ \\
\hline Bacillus atrophaeus & $\mathrm{nr}$ & UHT milk & $\begin{array}{l}20 \mathrm{kHz} \text {, probe, } 216 \mu \mathrm{m}, \\
(500 \mathrm{~W}), 6 \mathrm{~mL}\end{array}$ & $\mathrm{nr}$ & TS & 84.8 & 5.8 & 2.0 & $\begin{array}{l}\text { Ganesan et al. } \\
2015\end{array}$ \\
\hline Geobacillus stearothermophilus & ATCC 7953 & $\begin{array}{l}\text { Milk (2.0\% fat)/ } \\
\text { Tryptic soy broth }\end{array}$ & $\begin{array}{l}20 \mathrm{kHz}, \text { probe, } \\
11 \mathrm{~W}, 5 \mathrm{~mL}\end{array}$ & 2.2 & TS & 73 & 2 & 0.2 & $\begin{array}{l}\text { Deshpande and Walsh } \\
2020\end{array}$ \\
\hline G. stearothermophilus & $\mathrm{nr}$ & $\begin{array}{l}\text { Skim milk } \\
\text { (31.5\% total solids) }\end{array}$ & $\begin{array}{l}20 \mathrm{kHz} \text {, probe, } 240 \mu \mathrm{m}, \\
34.7 \mathrm{~W}, 6 \mathrm{~mL}\end{array}$ & 5.8 & TS & 60 & 0.3 & 0.3 & $\begin{array}{l}\text { Beatty and Walsh } \\
2016\end{array}$ \\
\hline G. stearothermophilus & ATCC 15952 & Non-fat milk & $\begin{array}{l}20 \mathrm{kHz} \text {, probe, } 3 \mathrm{~s} \text { on } / 3 \mathrm{~s} \text { off, } \\
91.2 \mu \mathrm{m}, 73.6 \mathrm{~W}, 20 \mathrm{~mL}\end{array}$ & 3.7 & US & Ice bath & 10 & 0.3 & $\begin{array}{l}\text { Khanal et al. } \\
2014\end{array}$ \\
\hline Geobacillus sp. & $\mathrm{nr}$ & Distilled water & $20 \mathrm{kHz}$, probe, $8 \mathrm{~W}, 5 \mathrm{~mL}$ & 1.6 & US & Room T & 20 & 1.5 & $\begin{array}{l}\text { Palanisamy et al. } \\
2019\end{array}$ \\
\hline Bacillus coagulans & ATCC 12245 & Non-fat milk & $\begin{array}{l}20 \mathrm{kHz} \text {, probe, } 3 \mathrm{~s} \text { on } / 3 \mathrm{~s} \text { off, } \\
91.2 \mu \mathrm{m}, 73.6 \mathrm{~W}, 20 \mathrm{~mL},\end{array}$ & 3.7 & US & Ice bath & 10 & 0.2 & $\begin{array}{l}\text { Khanal et al. } \\
2014\end{array}$ \\
\hline Anoxybacillus flavithermus & TNO-09.006 & $\begin{array}{l}\text { Milk (2.0\% fat)/ } \\
\text { Tryptic soy broth }\end{array}$ & $\begin{array}{l}20 \mathrm{kHz}, \text { probe, } \\
11 \mathrm{~W}, 5 \mathrm{~mL}\end{array}$ & 2.2 & TS & 73 & 2 & 0.2 & $\begin{array}{l}\text { Deshpande and Walsh } \\
2020\end{array}$ \\
\hline A. flavithermus & $\mathrm{nr}$ & Distilled water & $20 \mathrm{kHz}$, probe, $8 \mathrm{~W}, 5 \mathrm{~mL}$ & 1.6 & US & Room T & 20 & 1.3 & $\begin{array}{l}\text { Palanisamy et al. } \\
2019\end{array}$ \\
\hline High-acid foods & & & & & & & & & \\
\hline Alicyclobacillus acidoterrestris & CCT 4384 & $\begin{array}{l}\text { Commercial apple juice } \\
\left(11^{\circ} \text { Brix, } \mathrm{pH} 3.3\right)\end{array}$ & $\begin{array}{l}35 \mathrm{kHz} \text {, bath, }(480 \mathrm{~W}) \text {, } \\
25 \mathrm{~mL}\end{array}$ & $<19.2$ & $\begin{array}{l}\text { TS } \\
\text { US }\end{array}$ & $\begin{array}{l}95 \\
\text { Room T }\end{array}$ & $\begin{array}{l}20 \\
20\end{array}$ & $\begin{array}{l}5.0 \\
<1.0\end{array}$ & $\begin{array}{l}\text { Tremarin et al. } \\
2017\end{array}$ \\
\hline A. acidoterrestris & $\begin{array}{l}\text { CCT } 4384 \\
\text { (6-month old) }\end{array}$ & $\begin{array}{l}\text { Commercial apple juice } \\
\left(11^{\circ} \text { Brix, } \mathrm{pH} 3.3\right)\end{array}$ & $\begin{array}{l}35 \mathrm{kHz} \text {, bath, (480 W), } \\
25 \mathrm{~mL}\end{array}$ & $<19.2$ & $\begin{array}{l}\text { TS } \\
\text { US }\end{array}$ & $\begin{array}{l}95 \\
\text { Room T }\end{array}$ & $\begin{array}{l}20 \\
60\end{array}$ & $\begin{array}{l}5.5 \\
0.8\end{array}$ & $\begin{array}{l}\text { Tremarin et al. } \\
2019\end{array}$ \\
\hline A. acidoterrestris & $\begin{array}{l}\text { NZRM } 4447 \\
\text { (ATCC 49025) }\end{array}$ & $\begin{array}{l}\text { Orange juice } \\
\left(9.5^{\circ} \text { Brix, } \mathrm{pH} 3.8\right)\end{array}$ & $\begin{array}{l}24 \mathrm{kHz} \text {, probe, } 125 \mu \mathrm{m} \text {, } \\
162 \mathrm{~W}, 8 \mathrm{~mL}\end{array}$ & 20.2 & TS & 78 & 30 & 1.0 & $\begin{array}{l}\text { Evelyn and Silva } \\
2016\end{array}$ \\
\hline A. acidoterrestris & ATCC 49025 & $\begin{array}{l}\text { Apple juice } \\
\left(11.8^{\circ} \text { Brix, pH 3.4) }\right.\end{array}$ & $\begin{array}{l}20 \mathrm{kHz} \text {, probe, } 95.2 \mu \mathrm{m}, \\
(480 \mathrm{~W}), 100 \mathrm{~mL}\end{array}$ & $<4.8$ & $\begin{array}{l}\text { US } \\
\text { US }\end{array}$ & $\begin{array}{l}44 \\
30\end{array}$ & $\begin{array}{l}30 \\
30\end{array}$ & $\begin{array}{l}0.0 \\
0.0\end{array}$ & $\begin{array}{l}\text { Ferrario et al. } \\
2015\end{array}$ \\
\hline
\end{tabular}

Nr- not reported.

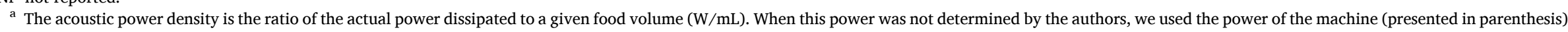
For the calculation and presented the power density with $<$ sign.

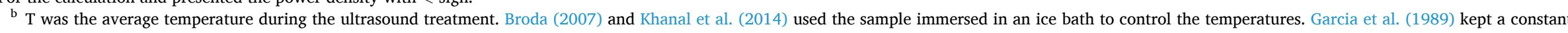

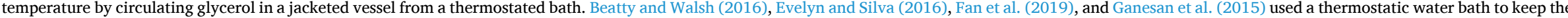

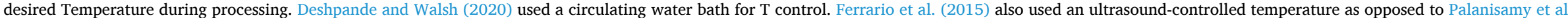

(2019). Tremarin et al. $(2017,2019)$ did not mention about the temperature control of ultrasound. 
Table 3

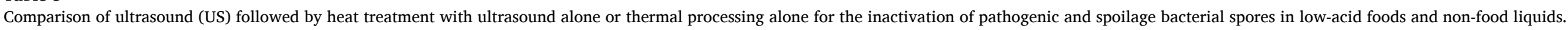

\begin{tabular}{|c|c|c|c|c|c|c|c|c|c|c|c|}
\hline \multirow[b]{2}{*}{ Species } & \multirow[b]{2}{*}{ Strains } & \multirow[b]{2}{*}{ Medium } & \multicolumn{4}{|l|}{ Ultrasound Processing } & \multicolumn{2}{|c|}{ Thermal Processing } & \multirow{3}{*}{$\frac{\frac{\text { Type }}{\text { of }}}{\text { Treatment }^{\mathrm{b}}}$} & \multirow[b]{2}{*}{ Log } & \multirow[b]{2}{*}{ Reference } \\
\hline & & & Ultrasound & Acoustic Power Density & $\mathrm{T}$ & Time & $\mathrm{T}$ & Time & & & \\
\hline & & Processed & Conditions & $(\mathrm{W} / \mathrm{mL})^{\mathrm{a}}$ & $\left({ }^{\circ} \mathrm{C}\right)$ & $(\min )$ & $\left({ }^{\circ} \mathrm{C}\right)$ & $(\min )$ & & Reduction & \\
\hline \multicolumn{12}{|l|}{ Pathogenic bacteria } \\
\hline \multirow[t]{2}{*}{ Clostridium perfringens } & 1362 & Distilled water & (75 W), probe, $20 \mathrm{~mL}$ & $<3.8$ & Ice bath & 12 & 90 & 30 & US $\rightarrow \mathrm{T}$ & $<1.0$ & Goodenough and Solberg \\
\hline & & & & - & & & 90 & 30 & $T$ & $<1.0$ & 1972 \\
\hline \multirow[t]{3}{*}{ Bacillus cereus } & $\mathrm{nr}$ & Diluted Ringer & $20 \mathrm{kHz}$, probe, $(60 \mathrm{~W})$, & $<15$ & $10-12$ & 12 & 110 & 15 & US $\rightarrow \mathrm{T}$ & 5.0 & Burgos et al. \\
\hline & & solution & $4 \mathrm{~mL}$ & & $10-12$ & 12 & & & US & 0.5 & 1972 \\
\hline & & & & & & & 110 & 15 & $T$ & $<2.0$ & \\
\hline \multirow[t]{3}{*}{ Bacillus licheniformis } & ATCC 6634 & Non-fat milk & $20 \mathrm{kHz}$, probe, $3 \mathrm{~s}$ on $/ 3 \mathrm{~s}$ off, & 3.7 & Ice bath & 10 & 63 & 30 & US $\rightarrow \mathrm{T}$ & 0.2 & Khanal et al. \\
\hline & & & $73.6 \mathrm{~W}, 20 \mathrm{~mL}$ & & Ice bath & 10 & & & us & 0.2 & 2014 \\
\hline & & & & & & & 63 & 30 & $T$ & 0.0 & \\
\hline \multirow[t]{3}{*}{ B. licheniformis } & $\mathrm{nr}$ & Diluted Ringer & $20 \mathrm{kHz}$, probe, & $<15$ & $10-12$ & 12 & 99 & 15 & US $\rightarrow \mathrm{T}$ & $\sim 3.5$ & Burgos et al. \\
\hline & & solution & $(60 \mathrm{~W}), 4 \mathrm{~mL}$ & & $10-12$ & 12 & & & uS & 0.2 & 1972 \\
\hline & & & - & - & & & 99 & 15 & $T$ & 2.5 & \\
\hline \multicolumn{12}{|l|}{ Spoilage bacteria } \\
\hline \multirow[t]{2}{*}{ Geobacillus stearothermophilus } & ATCC & Non-fat milk & $20 \mathrm{kHz}$, probe, $3 \mathrm{~s}$ on $/ 3 \mathrm{~s}$ off, & 3.7 & Ice bath & 10 & 80 & 1 & US $\rightarrow \mathrm{T}$ & 0.6 & Khanal et al. \\
\hline & 15,952 & & $73.6 \mathrm{~W}, 20 \mathrm{~mL}$ & & Ice bath & 10 & & & us & 0.3 & 2014 \\
\hline \multirow[t]{6}{*}{ Bacillus subtilis } & ATCC 6633 & Rice porridge & $20 \mathrm{kHz}$, probe, & 1.1 & Room T & 5 & 100 & 5 & $\mathrm{US} \rightarrow \mathrm{T}$ & 1.1 & Ansari et al. \\
\hline & & Whole milk & $114 \mu \mathrm{m}, 100 \mathrm{~mL}$ & & Room T & 5 & 100 & 5 & US $\rightarrow \mathrm{T}$ & 1.7 & 2017 \\
\hline & & Water & & & Room T & 5 & 100 & 5 & US $\rightarrow \mathrm{T}$ & 2.0 & \\
\hline & & Rice porridge & - & - & & & 100 & 5 & $T$ & 1.1 & \\
\hline & & Whole milk & - & - & & & 100 & 5 & $T$ & 1.2 & \\
\hline & & Water & - & - & & & 100 & 5 & $T$ & 1.6 & \\
\hline \multirow[t]{4}{*}{ B. subtilis } & 189 & Diluted Ringer & $20 \mathrm{kHz}$, probe, & $<12$ & Ice bath & 10 & 105 & 10 & US $\rightarrow \mathrm{T}$ & $<1.0$ & Ordonez and Burgos \\
\hline & & solution & $(60 \mathrm{~W}), 5 \mathrm{~mL}$ & & Ice bath & 10 & 110 & 10 & $\mathrm{US} \rightarrow \mathrm{T}$ & 2.5 & 1976 \\
\hline & & & - & - & & & 105 & 10 & $T$ & $<1.0$ & \\
\hline & & & - & - & & & 110 & 10 & $T$ & 2.0 & \\
\hline \multirow[t]{6}{*}{ B. subtilis } & var niger-40 & Whole milk & $20 \mathrm{kHz},(150 \mathrm{~W}), 30 \mathrm{~mL}$ & $<5$ & 31 & 15 & 100 & 10 & US $\rightarrow T$ & 1.5 & Garcia et al. \\
\hline & ATCC 6051 & & & & 31 & 15 & 100 & 10 & US $\rightarrow$ T & 3.6 & 1989 \\
\hline & var niger-40 & & & & 100 & 10 & - & - & $T S$ & 5.5 & \\
\hline & ATCC 6051 & & & & 100 & 10 & - & - & $T S$ & 6.3 & \\
\hline & var niger-40 & & - & - & & & 100 & 10 & $T$ & 0.9 & \\
\hline & ATCC 6051 & & - & - & & & 100 & 10 & $T$ & 3.9 & \\
\hline \multirow[t]{3}{*}{ Bacillus coagulans } & ATCC & Non-fat milk & $20 \mathrm{kHz}$, probe, $3 \mathrm{~s}$ on $/ 3 \mathrm{~s}$ off, & 3.7 & Ice bath & 10 & 63 & 30 & $\mathrm{US} \rightarrow \mathrm{T}$ & 0.3 & Khanal et al. \\
\hline & 12,245 & & $73.6 \mathrm{~W}, 20 \mathrm{~mL}$ & & Ice bath & 10 & & & US & 0.2 & 2014 \\
\hline & & & - & - & & & 63 & 30 & $T$ & 0.1 & \\
\hline
\end{tabular}

nr- not reported.

or the calculation and presented the power density with $<$ sign.

${ }^{\mathrm{b}}$ US $\rightarrow \mathrm{T}$, TS, US and T represent ultrasound followed by heat treatment, simultaneous ultrasound and heat treatment or thermosonication, ultrasound alone and thermal treatment alone, respectively. 
Table 4

Mold and yeast spore inactivation and modelling in high-acid foods and non-food liquids by thermosonication (TS).

\begin{tabular}{|c|c|c|c|c|c|c|c|c|c|}
\hline & & Acoustic & & & & & & & \\
\hline \multirow[t]{2}{*}{ Species } & \multirow{2}{*}{$\begin{array}{l}\text { Medium } \\
\text { Processed }\end{array}$} & \multirow{2}{*}{$\begin{array}{l}\text { Ultrasound } \\
\text { Conditions }\end{array}$} & \multirow{2}{*}{$\frac{\text { Power Density }}{(\mathrm{W} / \mathrm{mL})}$} & \multirow{2}{*}{$\frac{\text { Type of }}{\text { Treatment }}$} & \multirow{2}{*}{$\frac{\mathrm{T}^{\mathrm{a}}}{\left({ }^{\circ} \mathrm{C}\right)}$} & \multirow{2}{*}{$\frac{\text { Time }}{(\min )}$} & \multirow{2}{*}{$\frac{\text { Log }}{\text { reduction }}$} & Model and & \multirow[t]{2}{*}{ Reference } \\
\hline & & & & & & & & parameters $^{\mathrm{b}}$ & \\
\hline $\begin{array}{l}\text { Neosartorya fischeri } \\
\text { JCM } 1740 \text { (ATCC 1020) }\end{array}$ & \multirow[t]{2}{*}{$\begin{array}{l}\text { Apple juice } \\
\left(10.6^{\circ} \text { Brix, } \mathrm{pH} 3.7\right)\end{array}$} & \multirow[t]{2}{*}{$\begin{array}{l}24 \mathrm{kHz}, \text { probe, } \\
33 \mathrm{~W}, 100 \mathrm{~mL}\end{array}$} & \multirow[t]{2}{*}{0.33} & \multirow[t]{2}{*}{ TS } & \multirow[t]{2}{*}{75} & $0-70$ & Activation issues during the first $20 \mathrm{~min}$ & \multirow{2}{*}{$\begin{array}{l}\text { Lorentzian } \\
a=-2.1 ; b=5.9 ; c=22.4 ; d=24.8\end{array}$} & \multirow[t]{2}{*}{$\begin{array}{l}\text { Evelyn and Silva } \\
2017\end{array}$} \\
\hline (12-week old) & & & & & & 65 & 0.5 & & \\
\hline $\begin{array}{l}\text { Neosartorya fischeri } \\
\text { JCM } 1740 \text { (ATCC 1020) }\end{array}$ & \multirow[t]{2}{*}{$\begin{array}{l}\text { Apple juice } \\
\left(10.6^{\circ} \text { Brix, pH } 3.7\right)\end{array}$} & \multirow[t]{2}{*}{$\begin{array}{l}24 \mathrm{kHz}, \text { probe, } \\
33 \mathrm{~W}, 100 \mathrm{~mL}\end{array}$} & \multirow[t]{2}{*}{0.33} & \multirow[t]{2}{*}{ TS } & \multirow[t]{2}{*}{75} & $0-20$ & Activation issues during the first $10 \mathrm{~min}$ & \multirow[t]{2}{*}{$\begin{array}{l}\text { Lorentzian } \\
a=-6.1 ; b=8.5 ; c=11.5 ; d=18.6\end{array}$} & \multirow[t]{2}{*}{$\begin{array}{l}\text { Evelyn et al. } \\
2016\end{array}$} \\
\hline (4-week old) & & & & & & 25 & 0.5 & & \\
\hline $\begin{array}{l}\text { Byssochlamys nivea } \\
\text { JCM } 12806 \text { (CBS 696.95) }\end{array}$ & \multirow[t]{2}{*}{$\begin{array}{l}\text { Strawberry puree } \\
\left(8.1^{\circ} \text { Brix, } \mathrm{pH} 3.4\right)\end{array}$} & \multirow[t]{2}{*}{$\begin{array}{l}24 \mathrm{kHz}, \text { probe, } \\
33 \mathrm{~W}, 100 \mathrm{~mL}\end{array}$} & \multirow[t]{2}{*}{0.33} & \multirow[t]{2}{*}{ TS } & \multirow[t]{2}{*}{75} & $0-60$ & Activation issues during the first $7 \mathrm{~min}$ & \multirow{2}{*}{$\begin{array}{l}\text { Lorentzian } \\
a=-1.7 ; b=3.1 ; c=8.8 ; d=10.7\end{array}$} & \multirow[t]{2}{*}{$\begin{array}{l}\text { Evelyn and Silva } \\
2017\end{array}$} \\
\hline (12-week old) & & & & & & 60 & 1.8 & & \\
\hline Byssochlamys nivea & \multirow{2}{*}{$\begin{array}{l}\text { Strawberry puree } \\
\left(8.1^{\circ} \text { Brix, } \mathrm{pH} 3.4\right)\end{array}$} & \multirow{2}{*}{$\begin{array}{l}24 \mathrm{kHz}, \text { probe, } \\
33 \mathrm{~W}, 100 \mathrm{~mL}\end{array}$} & \multirow[t]{2}{*}{0.33} & \multirow[t]{2}{*}{ TS } & 75 & $0-10$ & Activation issues during the first $5 \mathrm{~min}$ & Lorentzian & Evelyn and Silva \\
\hline $\begin{array}{l}\text { JCM } 12806 \text { (CBS 696.95) } \\
\text { (4-week old) }\end{array}$ & & & & & & 15 & 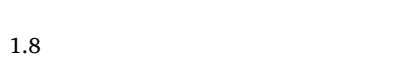 & $a=-9.1 ; b=10.1 ; c=5.1 ; d=15.9$ & $2015 c$ \\
\hline Aspergillus flavus & Sabouraud broth & $20 \mathrm{kHz}$, probe, & $\mathrm{nr}$ & TS & & & & First order & López-Malo et al. \\
\hline ATCC 16872 & $(\mathrm{pH} 3.0)$ & $120 \mu \mathrm{m}$ & & & 60 & 10 & 4.6 & $D_{T}=2.18 \mathrm{~min} ; z$-value $=17.3^{\circ} \mathrm{C}$ & 2005 \\
\hline & & & & & 52.5 & 10 & 1.7 & $D_{T}=5.9 \mathrm{~min}$ & \\
\hline Penicillium digitatum & Sabouraud broth & $20 \mathrm{kHz}$, probe, & $\mathrm{nr}$ & TS & & & & First order & López-Malo et al. \\
\hline LMU- DLA-2 & $(\mathrm{pH} 3.0)$ & $120 \mu \mathrm{m}$ & & & 52.5 & 10 & 1.6 & $D_{T}=6.31 \mathrm{~min} ; z$-value $=11.9{ }^{\circ} \mathrm{C}$ & 2005 \\
\hline Saccharomyces cerevisiae & Beer $(4.8 \%$ alc/vol $)$ & $24 \mathrm{kHz}$, probe, & 16.2 & TS & 55 & 10 & 3.1 & Weibull & Milani and Silva \\
\hline DSMZ 1848 & & $162 \mathrm{~W}, 10 \mathrm{~mL}$ & & & & & & $b=1.67 ; n=0.26$ & 2017 \\
\hline & Beer ( $0 \%$ alc/vol) & & 16.2 & TS & 55 & 10 & 3.3 & $b=2.66 ; n=0.11$ & \\
\hline
\end{tabular}

nr- not reported.

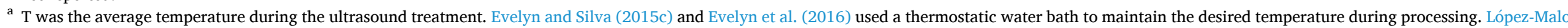
et al. (2005) controlled the temperature of the sample by circulating water with a refrigerated bath while Milani and Silva (2017) used a temperature-controlled jacketed vessel.

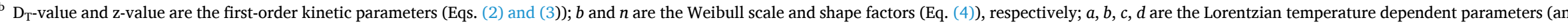

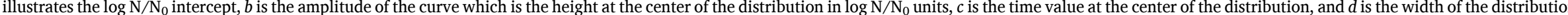
in time units (Eq. (5)). 
spores. To date, there are a limited number of studies on TS inactivation of pathogenic and spoilage bacteria in various foods and with different ultrasound intensities. Recently, other authors studied the impact of ultrasonication combined with heat $\left(80^{\circ} \mathrm{C}\right.$ for $\left.10 \mathrm{~min}\right)$ or preservatives (e.g. $0.5-1.0 \%$ nisin) on the germination and growth of spoilage/pathogenic bacterial spores in milk (Fan, Ismail, Hou, Muhammad, Ding, et al., 2019; Kakagianni, Chatzitzik, Koutsoumanis, \& Valdramidis, 2020), in which the inhibitory effect on growth was observed for the heat stress as opposed to the nisin preservative.

\section{Ultrasonication followed by heat treatment: effectiveness on bacterial spore inactivation}

Table 3 shows a summary of Clostridium and Bacillus spores' inactivation (in terms of log reductions) in which a sequential treatment of ultrasound followed by thermal treatment (US $\rightarrow \mathrm{T}$ ) was attempted to enhance the lethality of the treatment. The authors compared the log reductions achieved with US $\rightarrow \mathrm{T}$ treatment with the thermal treatment without US (T). Several authors also compared US $\rightarrow \mathrm{T}$ with US, and Garcia et al. (1989) also compared the sequential US $\rightarrow$ T treatment with TS and T. Ultrasound conditions with power density of $1.1-3.7 \mathrm{~W} / \mathrm{mL}$ during 5-15 min were applied to liquid or semisolid samples, followed by heating at temperatures in the range of $63-110{ }^{\circ} \mathrm{C}$ for $1-30 \mathrm{~min}$. Khanal et al. (2014) obtained almost no inactivation and no difference $(0.2-0.3)$ between US $\rightarrow \mathrm{T}\left(3.7 \mathrm{~W} / \mathrm{mL}-10 \mathrm{~min}\right.$ followed by $63{ }^{\circ} \mathrm{C}$ for 30 $\mathrm{min}$ ) or US and $\mathrm{T}$ alone at the same conditions for $B$. licheniformis and $B$. coagulans in non-fat milk. The same authors reported $0.6 \log$ for G. stearothermophilus after US $\rightarrow \mathrm{T}\left(3.7 \mathrm{~W} / \mathrm{mL}-10 \mathrm{~min}\right.$ followed by $80^{\circ} \mathrm{C}$ for $1 \mathrm{~min}$ ) vs 0.3 for US alone. Conclusively, these results show there are no major differences in the efficacy of these treatments (US $\rightarrow \mathrm{T}$, US and T).

On the contrary, Burgos et al. (1972) showed that US $\rightarrow$ T instead of thermal processing alone increased the $\log$ reductions of $B$. cereus from $<2.0$ to 5.0 ( $12 \mathrm{~min}$ US $\rightarrow 110^{\circ} \mathrm{C}-15 \mathrm{~min}$ ), although the difference was lower for B. licheniformis in diluted Ringer solution. Garcia et al. (1989) also compared US $\rightarrow \mathrm{T}\left(31^{\circ} \mathrm{C}-15 \mathrm{~min} \rightarrow 100{ }^{\circ} \mathrm{C}-10 \mathrm{~min}\right)$ and $\mathrm{T}$ effect on B. subtilis spores in whole milk and obtained a small increase (from 0.9 to 1.5) in the log reduction for var niger-40 strain, while the other strain ATCC 6051 showed no benefit from the sequential treatment. Although there was no difference in $B$. subtilis inactivation after US $\rightarrow \mathrm{T}$ ( $10 \mathrm{~min}$ US $\rightarrow 105{ }^{\circ} \mathrm{C}-10 \mathrm{~min}$ ) and $\mathrm{T}$ treatment alone in diluted Ringer solution, Ordonez and Burgos (1976) observed 0.5-1.0 log higher reductions of this bacteria at higher temperatures $\left(110{ }^{\circ} \mathrm{C}\right.$ for $\left.10 \mathrm{~min}\right)$, indicating a benefit from employing higher temperatures.

Garcia et al. (1989) compared TS to US $\rightarrow \mathrm{T}$ and $\mathrm{T}$ alone in which $\mathrm{T}$ and TS treatments were both conducted at $100{ }^{\circ} \mathrm{C}$, and registered much higher reductions of two strains of B. subtilis ( 5.5 and $6.3 \mathrm{log}$ ) with TS than US $\rightarrow$ T ( 1.5 and $3.6 \mathrm{log}$ ) or exclusive thermal treatment T (0.9 and $3.9 \mathrm{log}$ ). In general, it seemed that simultaneous application of ultrasound and heat or TS was better than the sequential application of treatments (US $\rightarrow \mathrm{T}$ ), as it was able to sensitize more the bacterial spores causing the decrease of their resistance $(>5.0 \mathrm{log}$ ) (Table 3). Higher inactivation for TS than for sequential ultrasound and heat indicates a synergistic bactericidal effect on spores of simultaneous application of both treatments. One possible explanation is the nonlethal intracellular injuries with ultrasound process, causing an increase of spores' sensitivity to heat treatment with subsequent disruption (Wordon, Mortimer, \& Mcmaster, 2012).

Depending on the bacteria or food the sequential treatment can present advantages when compared to thermal treatment alone (T). Single $\mathrm{T}$ was the least effective method for spore inactivation in foods. More studies conducted with different microbial species/strains, foods, ultrasound intensities and higher TS temperatures (between 90 and $100{ }^{\circ} \mathrm{C}$ ) are needed to compare the three ultrasound methods and draw more robust conclusions.

\section{Ultrasound and thermosonication inactivation of mold and yeast spores}

\subsection{Mold and yeast spore-formers}

Mold and yeast spore-formers are primary contaminants of high-acid and acidified drinks. Heat-resistant molds belong to Byssochlamys (Byssochlamys nivea, Byssochlamys fulva), Neosartorya (Neosartorya fischeri), Talaromyces (Talaromyces avellanus, Talaromyces flavus, Talaromyces bacillisporus, Talaromyces trachyspermus), Eurotium (Eurotium repens, Eurotium amstelodami, Eurotium chevalieri, and E. rubrum), Eupenicillium genera (Penicillium expansum, Penicillium digitatum, Eupenicillium javanicum, Eupenicillium brefeldianum) and Aspergillus (Aspergillus flavus, Aspergillus ochraceus, Aspergillus tamarii) (Abdel-Azeem et al., 2016; Pitt \& Hocking, 2009; Rao and Ramalakshmi, 2011; Salomão, 2018; Silva \& Evelyn, 2018; Silva \& Gibbs, 2009; Tournas, 1994) are typical species associated with spoilage of these drinks during distribution at room temperature or under refrigerated conditions (Silva \& Evelyn, 2020).

Saccharomyces (Saccharomyces cerevisiae, Saccharomyces bisporus), Zygosaccharomyces (Zygosaccharomyces bailii, Zygosaccharomyces rouxii) (Pitt \& Hocking, 2009; Silva \& Evelyn, 2018), Pichia (Pichia membranaefaciens, Pichia anomala) (Deák \& Beuchat, 1993; Pitt \& Hocking, 2009) and Rhodotorula (Rhodotorula mucilaginosa, Rhodotorula rubra) (Deák \& Beuchat, 1993) are among the most common heat-resistant yeast species in high-acid and acidified foods. S. cerevisiae and Pichia membranefaciens were found in contaminated cheese and milk (Fleet, 1990).

\subsection{Effect of US and TS on molds and yeasts spores}

Table 4 presents the ultrasound and TS $(0.33$ and $16.2 \mathrm{~W} / \mathrm{mL}$, $55-75{ }^{\circ} \mathrm{C}$ ) inactivation of mold and yeast spores in high-acid foods and non-food liquids. Yeast spores were able to resist the US in $0 \%$ alc/vol beer, with $1.2 \log$ reductions after $16.2 \mathrm{~W} / \mathrm{mL}$ US process for $15 \mathrm{~min}$ (Milani \& Silva, 2017). TS at $75^{\circ} \mathrm{C}-0.33 \mathrm{~W} / \mathrm{mL}$ promoted a slow process of ascospore activation of 4-week old $N$. fischeri mold suspended in apple juice during the first $10 \mathrm{~min}$, followed by inactivation, thus only $0.5 \mathrm{log}$ reduction registered after $25 \mathrm{~min}$ TS process (Evelyn, Kim, \& Silva, 2016). The same activation issues were also observed with 4-week old B. nivea, another heat resistant mold which was tested in strawberry puree during the first 5 min (Evelyn \& Silva, 2015c). These activation of ascospores were followed by inactivation $(0.5 \log$ for $N$. fischeri after 25 min and $1.8 \log$ for B. nivea after $15 \mathrm{~min}$ ) (Evelyn et al., 2016; Evelyn \& Silva, 2015c). Increasing the age of these ascospores to 12 weeks, also increased the time needed to inactivate the spores $(0.5 \log$ for $N$. fischeri after $65 \mathrm{~min}$ and $1.8 \mathrm{log}$ for B. nivea after $60 \mathrm{~min}$ ) (Evelyn et al., 2016; Evelyn \& Silva, 2015c). The increase in resistance of older ascospores has been correlated with a more robust structure of the spore cell wall (Evelyn \& Silva, 2017; Sussman \& Halvorson, 1966; Wyatt et al., 2015). Other investigators concluded TS at $52.5^{\circ} \mathrm{C}$ for $10 \mathrm{~min}$ with A. flavus and $P$. digitatum, resulted in lower inactivation levels $(1.6-1.7 \log )$ than TS at $60{ }^{\circ} \mathrm{C}$ in Sabouraud broth (López-Malo, Palou, Jiménez-Fernández, Alzamora, \& Guerrero, 2005). S. cerevisiae yeast spores seemed to be the least resistant presenting 3.1 and $3.3 \log$ reductions in $4.8 \%$ alc/vol and alcohol-free beers, respectively, after $16.2 \mathrm{~W} / \mathrm{mL}-55{ }^{\circ} \mathrm{C}-10 \mathrm{~min}$ (Milani \& Silva, 2017). Larger cells (e.g. yeast) have been considered more susceptible to ultrasound treatment than small cells, such as bacteria (Chemat et al., 2011). Nonetheless, each microbe has its specific structure which might respond differently to ultrasound processing (Gao et al., 2016).

Among all the molds and yeasts studied $N$. fischeri was the most TS resistant with a resistance comparable to bacteria. Older spores (12week old) of $N$. fischeri and B. nivea demonstrated higher resistance to TS, thus posing a concern due to spores' activation and very long treatment times ( $>60 \mathrm{~min}$ ) required for only 1-1.5 log reduction (Evelyn 
Table 5

Modelling the pathogenic and spoilage bacterial spore inactivation in foods and non-food liquids after thermosonication (TS) and ultrasound (US).

\begin{tabular}{|c|c|c|c|c|c|c|c|c|c|}
\hline & & & & Acoustic & & & & & \\
\hline \multirow[t]{2}{*}{ Species and Strain } & Medium & $\mathrm{pH}$ & Ultrasound & Power Density & Type of & $\mathrm{T}^{\mathrm{b}}$ & Model & Model parameters ${ }^{c}$ & Reference \\
\hline & Processed & & Conditions & $(\mathrm{W} / \mathrm{mL})^{\mathrm{a}}$ & Treatment & $\left({ }^{\circ} \mathrm{C}\right)$ & & & \\
\hline Clostridium perfringens & & & & & & & & & Evelyn and Silva \\
\hline NZRM 898 & Beef slurry & 6.5 & $24 \mathrm{kHz}$, probe, $210 \mu \mathrm{m}$, & 0.33 & TS & 75 & Weibull & $b=0.42 ; n=0.23$ & $2015 b$ \\
\hline NZRM 2621 & & & $33 \mathrm{~W}, 100 \mathrm{~g}$ & & TS & & & $b=0.18 ; n=0.61$ & \\
\hline Bacillus cereus & Beef slurry & 6.5 & $24 \mathrm{kHz}$, probe, $0.5 \mathrm{~s}$ on $/ 0.5 \mathrm{~s}$ off, & 0.33 & TS & 70 & First order & $D_{T}=0.36 \mathrm{~min} ; z$-value $=25.8^{\circ} \mathrm{C}$ & Evelyn and Silva \\
\hline NZRM 984 & & & $210 \mu \mathrm{m}, 33 \mathrm{~W}, 100 \mathrm{~mL}$ or $100 \mathrm{~g}$ & & US & 23 & & $D_{T}=140.90 \mathrm{~min}$ & $2015 a$ \\
\hline \multirow[t]{2}{*}{ (psychrotrophic) } & Skim milk & 6.5 & & 0.33 & TS & 70 & First order & $D_{T}=2.93 \mathrm{~min} ; z$-value $=45.7^{\circ} \mathrm{C}$ & \\
\hline & & & & & US & 23 & & $D_{T}=119.30 \mathrm{~min}$ & \\
\hline \multirow[t]{2}{*}{ B. cereus } & Distilled water & $\mathrm{nr}$ & $\begin{array}{l}20-60 \mathrm{kHz}, \text { probe, } 10 \mathrm{~s} \text { on } / 3 \mathrm{~s} \text { off, } \\
(300 \mathrm{~W}), 100 \mathrm{~mL} \text { or } 100 \mathrm{~g}\end{array}$ & $<3$ & TS & $50-70$ & $\begin{array}{l}\text { Second degree } \\
\text { polynomial } \\
\text { (RSM) }\end{array}$ & $\begin{array}{l}Y=0.9+0.028 f+0.012 t+0.096 T \\
-0.011 f t-0.016 \mathrm{fT}+0.008 t T-0.079 f^{2} \\
-0.055 t^{2}-0.10 T^{2}\end{array}$ & $\begin{array}{l}\text { Owusu-Ansah et al. } \\
2020\end{array}$ \\
\hline & Blended pork & $\mathrm{nr}$ & & $<3$ & TS & $50-70$ & $\begin{array}{l}\text { Second degree } \\
\text { polynomial } \\
\text { (RSM) }\end{array}$ & $\begin{array}{l}Y=0.57-0.001 f+0.017 t+0.074 T \\
-0.007 f t-0.006 \text { fT }+0.025 t T-0.094 f^{2} \\
-0.12 t^{2}-0.061 T^{2}\end{array}$ & \\
\hline $\begin{array}{l}\text { Bacillus subtilis } \\
\text { var niger-40 } \\
\text { ATCC } 6051\end{array}$ & Whole milk & $\mathrm{nr}$ & $20 \mathrm{kHz},(150 \mathrm{~W}), 30 \mathrm{~mL}$ & $<5$ & TS & 100 & First order & $\begin{array}{l}D_{T}=1.82 \mathrm{~min} ; z \text {-value }=6.3^{\circ} \mathrm{C} \\
D_{T}=1.60 \mathrm{~min} ; z \text {-value }=9.4^{\circ} \mathrm{C}\end{array}$ & $\begin{array}{l}\text { Garcia et al. } \\
1989\end{array}$ \\
\hline $\begin{array}{l}\text { Bacillus } \\
\text { atrophaeus }\end{array}$ & UHT milk & $\mathrm{nr}$ & $\begin{array}{l}20 \mathrm{kHz}, \text { probe, } 216 \mu \mathrm{m}, \\
0.64-11.12 \mathrm{~W}, 6 \mathrm{~mL}\end{array}$ & $0.11-1.85$ & TS & $0-72$ & $\begin{array}{l}\text { Second degree } \\
\text { polynomial } \\
\text { (RSM) }\end{array}$ & $\begin{array}{l}Y=-0.137-0.147 t+0.007 T+0.172 A \\
+0.036 t^{2}+0.0002 T^{2}-0.0018 \mathrm{TA}\end{array}$ & $\begin{array}{l}\text { Ganesan et al. } \\
2015\end{array}$ \\
\hline Geobacillus stearothermophilus & Skim milk & $\mathrm{nr}$ & $\begin{array}{l}20 \mathrm{kHz} \text {, probe, } 240 \mu \mathrm{m} \text {, } \\
17.54-34.79 \mathrm{~W}, 6 \mathrm{~mL}\end{array}$ & $2.9-5.8$ & TS & $45-75$ & $\begin{array}{l}\text { Second degree } \\
\text { polynomial } \\
\text { (RSM) }\end{array}$ & $\begin{array}{l}Y=0.66+0.0015 S+0.07 T-0.024 t \\
-0.16 S^{2}-0.085 T^{2}-0.065 T t-0.16 t^{2}\end{array}$ & $\begin{array}{l}\text { Beatty and Walsh } \\
2016\end{array}$ \\
\hline Alicyclobacillus acidoterrestris & $\begin{array}{l}\text { Commercial } \\
\text { apple juice }\end{array}$ & 3.3 & $\begin{array}{l}35 \mathrm{kHz} \text {, bath, }(480 \mathrm{~W}) \\
25 \mathrm{~mL}\end{array}$ & $<19.2$ & TS & 95 & First order & $D_{T}=8.9 \mathrm{~min}$ & $\begin{array}{l}\text { Tremarin et al. } \\
2017\end{array}$ \\
\hline CCT 4384 & $\left(11^{\circ} \mathrm{Brix}\right)$ & & & & US & Room T & & $D_{T}=69.8 \mathrm{~min}$ & \\
\hline $\begin{array}{l}\text { A. acidoterrestris } \\
\text { CCT } 4384\end{array}$ & $\begin{array}{l}\text { Commercial } \\
\text { apple juice }\end{array}$ & 3.3 & $\begin{array}{l}35 \mathrm{kHz} \text {, bath, (480 W), } \\
25 \mathrm{~mL}\end{array}$ & $<19.2$ & TS & 95 & Weibull & $\begin{array}{l}\beta=4.67 ; \alpha=1.18 \\
b=0.16 ; n=1.18\end{array}$ & $\begin{array}{l}\text { Tremarin et al. } \\
2019\end{array}$ \\
\hline (6-month-old) & $\left(11^{\circ}\right.$ Brix $)$ & & & & & & & & \\
\hline A. acidoterrestris & $\begin{array}{l}\text { Orange juice } \\
\left(9.5^{\circ} \text { Brix }\right)\end{array}$ & 3.8 & $\begin{array}{l}24 \mathrm{kHz}, \text { probe, } 125 \mu \mathrm{m} \text {, } \\
162 \mathrm{~W}, 8 \mathrm{~mL}\end{array}$ & 20.2 & TS & 78 & First order & $D_{T}=28.0 \mathrm{~min}$ & $\begin{array}{l}\text { Evelyn and Silva } \\
2016\end{array}$ \\
\hline
\end{tabular}

nr- not reported.

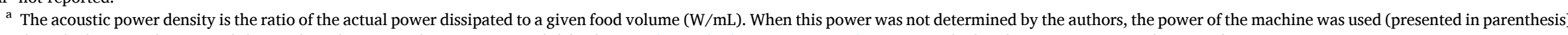
for the calculation and presented the result with < sign. The paste/semi-solid foods in Evelyn and Silva (2015a, 2015b) were weighed and energy is expressed as specific acoustic power (W/g).

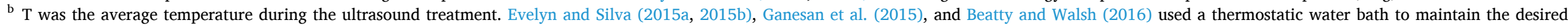
temperature during processing. Garcia et al. (1989) kept a constant by circulating glycerol from a thermostated bath. Tremarin et al. (2017, 2019) did not mention about the temperature control of ultrasound.

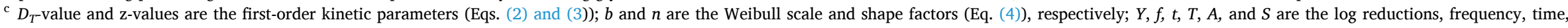

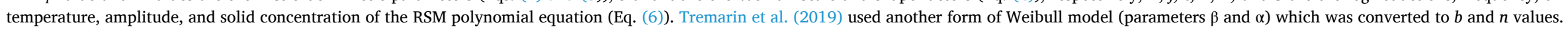


\& Silva, 2017). Temperature also plays a significant role in the inactivation by TS. To date, no data was found on the effect of TS or US on mold spores in low-acid foods, although contamination and spoilage with molds is also possible (Evelyn \& Silva, 2019).

\section{Mathematical models for US and TS inactivation of microbial spores}

\subsection{Inactivation kinetics and equations}

The inactivation behavior of microorganisms in foods under traditional thermal treatment or other non-thermal technologies such as TS is often considered as a first order, thus the simple first-order kinetics is the most common approach. The model has the following form (Eq. (1)):

$\log \frac{N}{N_{0}}=-k t$

with $N$ is the number of survivors after exposure to US or TS treatment for a specific time $\mathrm{t}(\mathrm{min}), N_{0}$ is the initial or untreated cell population in the food ( $\mathrm{cfu} / \mathrm{g}$ or $\mathrm{cfu} / \mathrm{mL}$ ), and $k$ is the first-order or inactivation rate constant $\left(\mathrm{min}^{-1}\right)$. This equation is commonly expressed by microbiologists as follows (Eq. (2)):

$\log \frac{N}{N_{0}}=-\frac{t}{D_{T}}$

from which $D_{T}$ values are estimated from Equation (2) (min) or calculated from $k(D=2.303 / k) . D_{T}$-value which is the time in min at a certain temperature $T$ necessary to cause one decimal reduction in the microbial population, $10^{\mathrm{n}}$ to $10^{\mathrm{n}-1}$ in the microbial concentration in food (Bigelow, 1921). The logarithm of $D$-values (obtained from the primary model) can be plotted against TS temperature (or US intensity), in which the reciprocal of the slope is equal to $z_{T}$-value $\left({ }^{\circ} \mathrm{C}\right)$ and defined as the temperature increase that results in a 10 -fold decrease in the $D$-value (Eq. (3)).

$z_{T}=\frac{T_{r e f}-T}{\log D-\log D_{T r e f}}$

where $D_{\text {Tref }}$ is the $D$-value at a reference temperature $T_{\text {ref }}\left({ }^{\circ} \mathrm{C}\right)$, and $T$ is the temperature of the isothermal treatment $\left({ }^{\circ} \mathrm{C}\right)$.

Weibull model is a good approach when the microbial log survivors at a specific TS temperature or intensity show a concavity or a tail. The two main parameters obtained are $b$ (the scale factor) and $n$ (the survival curve shape factor). This model is based on a vitalistic approach i.e. heterogeneity in the resistance distributed among individual cells within a population (Van Boekel, 2002) (Eq. (4)):

$\log \frac{N}{N_{0}}=-b t^{n}$

The scale factor is a rate parameter related to the velocity of inactivation of the microorganism, whereas the shape factor describes the degree of curvilinearity. $n<1, n>1$ and $n=1$ correspond to survival curves with concave-upwards (tailings) and convex or concavedownwards (shoulders), and the Weibull model becomes the same as a simple first-order kinetic model, respectively.

Lorentzian is a non-linear model which has been used to describe the log survivors of resistant molds with thermosonication (TS) treatment, showing an initial activation shoulder followed by approximately linear inactivation (Evelyn \& Silva, 2015c; Evelyn et al., 2016). The four parameter Lorentzian curve peak functions $(a, b, c, d)$ in Eq. (4) are obtained, where an illustrates the $\log \mathrm{N} / \mathrm{N}_{0}$ intercept; $b$ acts an amplitude of curve that is the height at the center of the distribution in log $\mathrm{N} / \mathrm{N}_{0}$ units; $c$ is the center that is the time $(\mathrm{t})$ value at the center of the distribution, and $d$ is the width, a measure of the width of the distribution in the same units as t (Lorentz, 1875; Peng \& Lu, 2006): $\log \frac{N}{N_{0}}=a+\frac{b}{1+\left(\frac{t-c}{d}\right)^{2}}$

Another possible approach is the polynomial model or response surface model (RSM), which is primarily an empirical model developed to predict the effect of multiple environmental factors (variables) on the microbial spore inactivation (Silva, Gibbs, Vieira, \& Silva, 1999). A second-order polynomial equation involving first-order, second-order (quadratic), and interaction terms is generally used (Eq. (6)):

$Y=B_{0}+\sum_{i=1}^{n} B_{i} X_{i}+\sum_{i=1}^{n} B_{i i} X_{i}^{2}+\sum_{j \neq 1}^{n} B_{i j} X_{i} X_{j}+\varepsilon$

where $Y$ is the microbial spore logarithmic reduction $\left(\log \mathrm{N} / \mathrm{N}_{0}\right) ; B_{0}, B_{i}$, $B_{i i}$, and $B_{i j}$ are the estimated regression coefficients; $X_{i}$ and $X_{j}$ are the environmental factors such as temperature and amplitude; and $\varepsilon$ is the error term. The RSM can be used to find the variables conditions resulting in maximum response $(Y)$ (Beatty \& Walsh, 2016; Ganesan et al., 2015).

To summarize, first-order and Weibull models (Equations (1), (2), (4) and (5)) can be used to characterize microbial spore inactivation for a single variable (fixed US/TS intensity or TS temperature). The Weibull model is often a more flexible model than the former one, as it is also applicable to non-linear survival curves, frequently showing downward and upward concavities (Van Boekel, 2002). As opposed to the first-order and Weibull models, the RSM model is able to include the effects of US/TS intensity, TS temperature and time in one single equation and predict conditions for maximum spore inactivation.

\subsection{Bacterial spores}

Table 5 shows the model parameters estimated for describing TS and US inactivation of bacterial spores. The survival curves of bacterial spores by US alone displayed linear inactivation, thus first-order kinetics (Eqs. (1)-(3)). For example, US $\left(0.33 \mathrm{~W} / \mathrm{g}\right.$ or $\left.\mathrm{W} / \mathrm{mL}-23^{\circ} \mathrm{C}\right)$ inactivation of psychrotrophic $B$. cereus spores resulted in $D$-values of $141 \mathrm{~min}$ and $119 \mathrm{~min}$ in beef slurry and skim milk, respectively (Evelyn \& Silva, 2015a). US $(<19.2 \mathrm{~W} / \mathrm{mL})$ inactivation of $A$. acidoterrestris at room $\mathrm{T}$ resulted in $D$-values of $70 \mathrm{~min}$ in apple juice (Tremarin et al., 2017). Similarly, TS inactivation of both species also demonstrated a linear trend, with the following $D$-values: TS at $0.33 \mathrm{~W} / \mathrm{g}$ or $\mathrm{W} / \mathrm{mL}$, $D_{70}{ }^{\circ} \mathrm{C}^{-v}$ alues $=0.36 \mathrm{~min}$ for $B$. cereus in beef slurry and $D_{70}{ }^{\circ} \mathrm{C}^{-v a l u e s}=$ $2.93 \mathrm{~min}$ for $B$. cereus in skim milk, with $z_{\mathrm{T}}$-values $=25.8-45.7{ }^{\circ} \mathrm{C}$

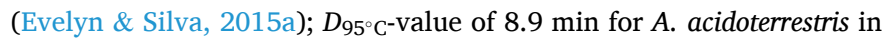

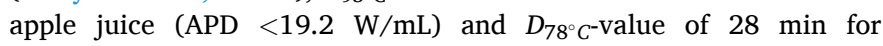
A. acidoterrestris in orange juice (APD $20.2 \mathrm{~W} / \mathrm{mL}$ ) (Evelyn \& Silva,

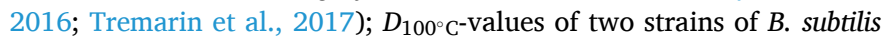
spores of $1.60-1.82 \mathrm{~min}$ in whole milk with $z_{\mathrm{T}}$-values within $6.3-9.4{ }^{\circ} \mathrm{C}$ for APD of $<5 \mathrm{~W} / \mathrm{mL}$ (Garcia et al., 1989).

As opposed to their past works, Tremarin et al. (2019) demonstrated that the Weibull model (Eq. (4)) fitted the downward concavity survival data better than the first-order kinetics in describing TS $(<19.2$ $\mathrm{W} / \mathrm{mL}-95^{\circ} \mathrm{C}$ ) inactivation of 6-month old A. acidoterrestris in apple juice with $n=1.18$. TS $\left(0.33 \mathrm{~W} / \mathrm{g}-75^{\circ} \mathrm{C}\right)$ inactivation of $C$. perfringens in beef slurry exhibited an upward concavity ( $n$ between 0.23 and 0.61 ) and was also best described by the Weibull model (Evelyn \& Silva, 2015b). These results suggest that the TS inactivation of Clostridium genera and older spores of $A$. acidoterrestris pose a challenge to ultrasound processors, thus harsher processing conditions (higher temperature and/or acoustic power density) might be more effective. Subsequently, there is a need to design ultrasound units withstanding high temperature as previously mentioned. Nonetheless, more experiments are still required to better elucidate the kinetics of bacterial spore inactivation by TS.

Response surface methodology has also been used by several authors to investigate the simultaneous effects of ultrasound frequency (f), time 
$(\mathrm{t})$, and temperature $(\mathrm{T})$ or time $(\mathrm{t})$, temperature $(\mathrm{T})$, and amplitude $(\mathrm{A})$ or solid concentration $(\mathrm{S})$, temperature $(\mathrm{T})$, and treatment time $(\mathrm{t})$ on spore inactivation, and if possible to predict the processing conditions to achieve a desired log reduction of spores (Eq. (6)). For example, the predicted optimum process conditions are $84.8^{\circ} \mathrm{C}-216 \mu \mathrm{m}-5.8 \mathrm{~min}$ for $2.0 \mathrm{log}$ reduction of $B$. atrophaeus spores in UHT milk, $31.5 \% \mathrm{~S}-60$ ${ }^{\circ} \mathrm{C}-0.3 \mathrm{~min}$ for $0.4 \log$ reduction of stearothermophilus spores in skim milk, and $20 \mathrm{~Hz}-70{ }^{\circ} \mathrm{C}-13.56 \mathrm{~min}$ for $0.5 \mathrm{log}$ reduction of $B$. cereus spores in pork (Beatty \& Walsh, 2016; Ganesan et al., 2015; Owusu-Ansah eta al., 2020), still revealing insufficient TS inactivation.

\subsection{Mold and yeast spores}

Table 4 shows the reports regarding TS inactivation kinetics of mold and yeast spores. Milani and Silva (2017) investigated the effect of US alone $\left(16.2 \mathrm{~W} / \mathrm{mL}-23{ }^{\circ} \mathrm{C}\right)$ on $S$. cerevisiae spore survival in $0-7.0 \%$ alc/vol beers, and the curves showed a concave upward trend with low inactivation levels ( $\leq 1.2$ log after $15 \mathrm{~min})$.

The first-order model has been used to describe the log survivors of spores of A. flavus and $P$. digitatum in Sabouraud broth after $120 \mu \mathrm{m}$ TS:

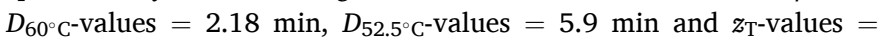

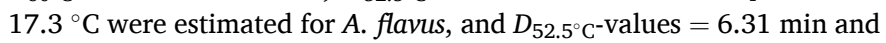
$z_{\mathrm{T}}$-values $=11.9{ }^{\circ} \mathrm{C}$ were obtained for $P$. digitatum (López-Malo et al., 2005). Milani and Silva (2017) reported Weibull model for TS (16.2 $\mathrm{W} / \mathrm{mL}-55^{\circ} \mathrm{C}$ ) inactivation of $S$. cerevisiae in $0 \%$ and $4.8 \%$ alc/vol beer due to an upward concavity ( $n$ between 0.11 and 0.26 ) observed in the survival curves, revealing an undesirable increase in spore resistance with processing time. The Lorentzian model was another non-linear model also used to describe the inactivation of the most resistant ( $N$. fischeri and B. nivea, 4 and 12-week old) mold spores, exhibiting survival curves with an activation shoulders followed by a linear inactivation of spores (Eq. (5)) (Evelyn et al., 2016; Evelyn \& Silva, 2015c). Similar to bacteria, these results also indicate that ultrasound TS inactivation of certain species of resistant mold/yeast spores could exhibit non-linearity (Weibull and Lorentzian models adjusted to spore log survival data).

\section{Conclusions and final remarks}

Some bacteria, molds and yeasts can produce spores, a more resistant form of the microorganism. Ultrasound (US) alone is unable to inactivate microbial spores. Thermosonication (TS) is a better method than US, followed by heat treatment or conventional thermal processing for the inactivation of spores in liquid or semisolid foods. TS at $\geq 95{ }^{\circ} \mathrm{C}$ is needed to obtain $5 \mathrm{D}$ reduction in spores, as TS temperature plays a significant role in spore inactivation. The magnitude of pathogenic and spoilage spore resistance to TS is similar. The TS inactivation of resistant mold spores, critical for high-acid fruit products, was difficult and demonstrated challenging behaviors. TS spore resistance is also affected by the type of cell (microbial species/strain) and the beverage, indicating the importance of testing the most resistant spores in a specific food to design successful TS pasteurization for that food. Linear and nonlinear trends were observed for bacterial, mold and yeast spore inactivation in foods and non-food liquids after TS treatments. Species and spore age affected the inactivation kinetics and the presence of a nonlinear inactivation pattern. The concave upward of the log survival curves of $C$. perfringens described by Weibull model can be translated in an increase of microbial spore resistance with TS processing time. Similarly, the activation shoulder exhibited by $N$. fischeri and B. nivea molds submitted to TS is a concern and should be interpreted with more care. Thus, TS at higher T should be attempted.

To summarize, uniformity in reporting the intensity of the TS/US process is needed, such as using the acoustic power density in $\mathrm{W} / \mathrm{mL}$, so that proper comparison of results from different research groups is possible. More experiments should be carried out using higher TS temperature or ultrasound acoustic power density, and a wider range of TS conditions. US probes withstanding temperatures close to $100{ }^{\circ} \mathrm{C}$ are required, as those will enable a more rapid pasteurization than a conventional thermal pasteurization process. However, this increase in TS temperature is limited by the increase in water vapor tension, which reduces the cavitation effect. The addition of an external pressure could increase the power of bubble implosion hence the microbial inactivation, thus manothermosonication technology could possibly improve pasteurization performance and subsequent food preservation.

\section{References}

Abdel-Azeem, A. M., Salem, F. M., Abdel-Azeem, M. A., Nafady, N. A., Mohesien, M. T., \& Soliman, E. A. (2016). Biodiversity of the genus Aspergillus in different habitats. In V. K. Gupta (Ed.), New and future developments in microbial biotechnology and bioengineering (pp. 3-28). Elsevier B. V.

Andersson, A., Ronner, U., \& Granum, P. E. (1995). What problems does the food industry have with the spore-forming Bacillus cereus and Clostridium perfringens? International Journal of Food Microbiology, 28, 145-155.

Ansari, J., Ismail, M., \& Farid, M. (2017). Investigation of the use of ultrasonication followed by heat for spore inactivation. Food and Bioproducts Processing, 104, 32-39.

Aureli, P., Fenica, L., \& Franciosa, G. (1999). Classic and emergent forms of botulism: The current status in Italy. Euro Surveillance, 4(1), 7.

Aureli, P., Fenicia, L., Pasolini, B., Gianfranceschi, M., McCroskey, L. M., \& Hatheway, C. L. (1986). Two cases of type E infant botulism caused by neurotoxigenic Clostridium butyricum in Italy. Journal of Infectious Diseases, 154(2), 207-211.

Awad, T. S., Moharram, H. A., Shaltout, O. E., Asker, D., \& Youssef, M. M. (2012). Applications of ultrasound in analysis, processing and quality control of food: A review. Food Research International, 48, 410-427.

Azizi, A., \& Ranganna, S. (1993). Spoilage organisms of canned acidified mango pulp and their relevance to thermal processing of acid foods. Journal of Food Science \& Technology, 30(4), 241-245.

Barash, J. R., Tang, T. W. H., \& Arnon, S. S. (2005). First case of infant botulism caused by Clostridium baratii type F in California. Journal of Clinical Microbiology, 43(8), 4280-4282.

Beatty, N. F., \& Walsh, M. K. (2016). Influence of thermosonication on Geobacillus stearothermophilus inactivation in skim milk. International Dairy Journal, 61, 10-17.

Bermúdez-Aguirre, D. (2017). Advances in thermo- and manothermosonication for microbial Inactivation. In D. Bermúdez-Aguirre (Ed.), Ultrasound: Advances for food processing and preservation (pp. 15-37). Academic Press.

Bigelow, W. (1921). The logarithmic nature of thermal death time curves. Journal of Infectious Diseases, 29(5), 528-536.

Broda, D. M. (2007). The effect of peroxyacetic acid-based sanitizer, heat and ultrasonic waves on the survival of Clostridium estertheticum spores in vitro. Letters in Applied Microbiology, 45, 336-341.

Brown, K. L. (2000). Control of bacterial spores. British Medical Bulletin, 56(1), 158-171.

Burgos, J., Ordonez, J., \& Sala, F. (1972). Effect of ultrasonic waves on the heat resistance of Bacillus cereus and Bacillus licheniformis spores. Journal of Applied Microbiology, 24, 497-498.

Butz, P., \& Tauscher, B. (2002). Emerging technologies: Chemical aspects. Food Research International, 35, 279-284.

Carlin, F., Girardin, H., Peck, M. W., Stringer, S. C., Barker, G. C., Martinez, A., et al. (2000). Research on factors allowing a risk assessment of spore-forming pathogenic bacteria in cooked chilled foods containing vegetables: A fair collaborative project. International Journal of Food Microbiology, 60(2), 17-135.

Carlin, F., Guinebretiere, M., Choma, C., Pasqualini, R., Braconnier, A., \& Nguyen-the, C. (2000). Spore-forming bacteria in commercial cooked, pasteurised and chilled vegetable purees. Food Microbiology, 17(2), 153-165.

CDC (Centers for Disease Control). (2000). Human ingestion of Bacillus anthraciscontaminated meat - Minnesota, august 2000. Morbidity and Mortality Weekly Report, 49(36).

Cerny, G., Duong, H. A., Hennlich, W., \& Miller, S. (2000). Alicyclobacillus acidoterrestris. Influence of oxygen content on growth in fruit juices. Food Australia, 52(7), 289-291.

Chandler, D. P., Brown, J., Bruckner-Lea, C. J., Olson, L., Posakony, G. J., Stults, J. R., et al. (2001). Continuous spore disruption using radially focused, high-frequency ultrasound. Analytical Chemistry, 73, 3784-3789.

Charoux, C. M. G., O’Donnell, C. P., \& Tiwari, B. K. (2017). Ultrasound processing and food quality. Ultrasound. In D. Bermudez-Aguirre (Ed.), Advances for food processing and preservation (pp. 215-235). Academic Press.

Chaturvedi, A., \& Shukla, S. (2015). Occurrence of Clostridium species in different dairy products and its associated health risk. International Journal of Recent Scientific Research, 2, 2827-2829.

Chemat, F., Zill-e-Huma, \& Khan, M. K. (2011). Applications of ultrasound in food technology: Processing, preservation and extraction. Ultrasonics Sonochemistry, 18, 813-835.

Chen, D. (2012). Applications of ultrasound in water and wastewater treatment. In D. Chen, S. Sharma, \& A. Mudhoo (Eds.), Handbook on application of ultrasound: Sonochemistry for sustainability (pp. 373-406). Boca Raton, US: CRC Press.

Cosentino, S., Mulargia, A. F., Pisano, B., Tuveri, P., \& Palmas, F. (1997). Incidence and biochemical characteristics of Bacillus flora in Sardinian dairy products. International Journal of Food Microbiology, 38, 235-238.

De-Jong, J. (1989). Spoilage of an acid food product by Clostridium perfringens, C. barati and C. butyricum. International Journal of Food Microbiology, 8(2), 121-132. 
Deák, T., \& Beuchat, L. R. (1993). Yeasts associated with fruit juice concentrates. Journal of Food Protection, 56, 777-782.

Deinhard, G., Blanz, P., Poralla, K., \& Altan, E. (1987). Bacillus acidoterrestris sp. nov., a new thermotolerant acidophile isolated from different soils. Systematic \& Applied Microbiology, 10, 47-53.

Deshpande, D. K., \& Walsh, M. K. (2020). Effect of thermosonication in a batch system on the survival of spore-forming bacteria. International Journal of Dairy Technology, 70, $1-8$.

Dijksterhuis, J. (2007). Heat-resistant ascospores. In J. Dijksterhuis, \& R. A. Samson (Eds.), Food mycology: A multifaceted approach to fungi and food. Boca Raton, FL: CRC Press.

Dolas, R., Saravanan, C., \& Kaur, B. P. (2019). Emergence and era of ultrasonic's in fruit juice preservation: A review. Ultrasonics Sonochemistry, 58, 104609.

Dufrenne, J., Bijwaard, M., Te Giffel, M., Beumer, R., \& Notermans, S. (1995). Characteristics of some psychrotrophic Bacillus cereus isolates. International Journal of Food Microbiology, 27(2), 175-183.

Duong, H. A., \& Jensen, N. (2000). Spoilage of iced tea by Alicyclobacillus. Food Australia, 52(7), 292.

Evelyn, Kim, H. J., \& Silva, F. V. M. (2016). Modeling the inactivation of Neosartorya fischeri ascospores in apple juice by high pressure, power ultrasound and thermal processing. Food Control, 59, 530-537.

Evelyn, \& Silva, F. V. M. (2015a). Thermosonication versus thermal processing of skim milk and beef slurry: Modeling the inactivation kinetics of psychrotrophic Bacillus cereus spores. Food Research International, 67, 67-74.

Evelyn, \& Silva, F. V. M. (2015b). Use of power ultrasound to enhance the thermal inactivation of Clostridium perfringens spores in beef slurry. International Journal of Food Microbiology, 206, 17-23.

Evelyn, \& Silva, F. V. M. (2015c). Inactivation of Byssochlamys nivea ascospores in strawberry puree by high pressure, power ultrasound and thermal processing. International Journal of Food Microbiology, 214, 129-136.

Evelyn, \& Silva, F. V. M. (2016). High pressure processing pretreatment enhanced the thermosonication inactivation of Alicyclobacillus acidoterrestris spores in orange juice. Food Control, 62, 365-372.

Evelyn, \& Silva, F. V. M. (2017). Resistance of Byssochlamys nivea and Neosartorya fischeri mould spores of different age to high pressure thermal processing and thermosonication. Journal of Food Engineering, 201, 9-16.

Evelyn, \& Silva, F. V. M. (2018a). Inactivation of pathogenic microorganisms in foods by high pressure processing. In V. R. Rai, \& J. A. Bai (Eds.), Food safety and protection (pp. 343-378). Boca Raton, FL: CRC Press.

Evelyn, \& Silva, F. V. M. (2018b). Differences in the resistance of microbial spores to thermosonication, high pressure thermal processing, and thermal treatment alone. Journal of Food Engineering, 222, 292-297.

Evelyn, \& Silva, F. V. M. (2019). Heat assisted HPP for the inactivation of bacteria, moulds, and yeast spores in foods: Log reductions and mathematical models. Trends in Food Science \& Technology, 88, 143-156.

Fan, L., Ismail, B. B., Hou, F., Muhammad, A. I., Ding, T., \& Liu, D. (2019). Ultrasound pretreatment enhances the inhibitory effects of nisin/carvacrol against germination, outgrowth and vegetative growth of spores of Bacillus subtilis ATCC6633 in laboratory medium and milk: Population and single-cell analysis. International Journal of Food Microbiology, 311, 108329.

Fan, L., Ismail, B. B., Hou, F., Muhammad, A. I., Zou, M., Ding, T., et al. (2019). Thermosonication damages the inner membrane of Bacillus subtilis spores and impels their inactivation. Food Research International, 125, 108514.

FDA (U. S. Food and Drug Administration). (2004). Guidance for industry: Juice HACCP hazards and controls guidance. https://www.fda.gov/regulatory-information/sear ch-fda-guidance-documents/guidance-industry-juice-hazard-analysis-critical-contro l-point-hazards-and-controls-guidance-first Accessed on 30 June 2020.

Feligini, M., Brambati, E., Panelli, S., Ghitti, M., Sacchi, R., Capelli, E., et al. (2014). Oneyear investigation of Clostridium spp. occurrence in raw milk and curd of Grana Padano cheese by the automated ribosomal intergenic spacer analysis. Food Control, 42, 71-77.

Feng, H., \& Yang, W. (2011). Ultrasonic processing. In J. P. Clark, C. Ritson, G. W. Smithers, M. A. Augustin, W. Ellefson, L. Zach, et al. (Eds.), Nonthermal processing technologies for food (pp. 135-154). US: Wiley-Blackwell.

Ferrario, M., Alzamora, S. M., \& Guerrero, S. (2015). Study of the inactivation of spoilage microorganisms in apple juice by pulsed light and ultrasound. Food Microbiology, 46, 635-642.

Fleet, G. H. (1990). Yeasts in dairy products. Journal of Applied Bacteriology, 68, 199-211.

Ganesan, B., Martini, S., Solorio, J., \& Walsh, M. K. (2015). Determining the effects of high intensity ultrasound on the reduction of microbes in milk and orange juice using response surface methodology. International Journal of Food Science, 350719. 2015.

Gao, S., Lewis, G., Ashokkumar, M., \& Hemar, Y. (2014). Inactivation of microorganisms by low-frequency high-power ultrasound: 1 . Effect of growth phase and capsule properties of the bacteria. Ultrasonics Sonochemistry, 21(1), 446-453.

Gao, S., Lewis, G., \& Hemar, Y. (2016). Ultrasonic inactivation of microorganisms. In M. Ashokkumar (Ed.), Handbook of ultrasonics and sonochemistry (pp. 1355-1381). Singapore: Springer.

Garcia, M. L., Burgos, J., Sanz, B., \& Ordonez, J. (1989). Effect of heat and ultrasonic waves on the survival of two strains of Bacillus subtilis. Journal of Applied Bacteriology, 67, 619-628.

Garde, S., Ávila, M., Gómez, N., \& Nuñez, M. (2013). Clostridium in late blowing defect of cheese: Detection, prevalence, effects and control strategies. In H. Castelli, \& L. du Vale (Eds.), Handbook on cheese: Production, chemistry and sensory properties (pp. 503-518). New York: Nova Science Publishers.
Goodenough, E. R., \& Solberg, M. (1972). A technique for producing large yields of vegetative cell-free refractile Clostridium perfringens spores of unaltered heat resistance. Applied Microbiology, 23(2), 429-430.

Gould, G. W. (1999). Sous vide foods: Conclusions of an ECFF botulinum working party. Food Control, 10(1), 47-51.

Hormazabal, V., \& Granum, P. E. (2007). Food poisoning associated with pumilacidinproducing Bacillus pumilus in rice. International Journal of Food Microbiology, 115(3), 319-324.

Jackson, S. G., Goodbrand, R. B., Ahmed, R., \& Kasatiya, S. (1995). Bacillus cereus and Bacillus thuringiensis isolated in a gastroenteritis outbreak investigation. Letters in Applied Microbiology, 21(2), 103-105.

Janstová, B., \& Lukásová, J. (2001). Heat resistance of Bacillus spp. spores isolated from cow's milk and farm environment. Acta Veterinaria Brno, 70, 179-184.

Kakagianni, M., Chatzitzik, C., Koutsoumanis, K. P., \& Valdramidis, V. P. (2020). The impact of high power ultrasound for controlling spoilage of Alicyclobacillus acidoterrestris: A population and a single spore assessment. Innovative Food Science \& Emerging Technologies, 64, 102405.

Keynan, A. (1969). The outgrowing bacterial endospore as a system for the study of cellular differentiation. Current Topics in Developmental Biology, 4, 1-36.

Khanal, S. N., Anand, S., \& Muthukumarappan, K. (2014). Evaluation of high-intensity ultrasonication for the inactivation of endospores of 3 Bacillus species in nonfat milk. Journal of Dairy Science, 97, 5952-5963.

Kim, M., Zahn, M., Reporter, R., Askar, Z., Green, N., Needham, M., et al. (2019). Outbreak of foodborne Botulism associated with prepackaged pouches of liquid herbal tea. Open Forum Infectious Disease, 6(2), ofz014.

Ledenbach, L. H., \& Marshall, R. T. (2009). Microbiological spoilage of dairy products. In W. Sperber, \& M. Doyle (Eds.), Compendium of the microbiological spoilage of foods and beverages (pp. 41-67). New York: Springer.

Lindström, M., Kiviniemi, K., \& Korkeala, H. (2006). Hazard and control of group II (nonproteolytic) Clostridium botulinum in modern food processing. International Journal of Food Microbiology, 108(1), 92-104.

López-Malo, A., Palou, E., Jiménez-Fernández, M., Alzamora, S. M., \& Guerrero, S. (2005). Multifactorial fungal inactivation combining thermosonication and antimicrobials. Journal of Food Engineering, 67(1-2), 87-93.

Lorentz, H. A. (1875). On the theory of reflection and refraction of light. PhD Thesis. Netherlands: University of Leiden.

Lv, R., Muhammad, A. I., Zou, M., Yu, Y., Fan, L., Zhou, J., et al. (2020). Hurdle enhancement of acidic electrolyzed water antimicrobial efficacy on Bacillus cereus spores using ultrasonication. Applied Microbiology and Biotechnology, 104, 4505-4513.

Lv, R., Zou, M., Chantapakul, T., Chen, W., Zhou, J., Ding, T., et al. (2019). Effect of ultrasonication and thermal and pressure treatments, individually and combined, on inactivation of Bacillus cereus spores. Applied Microbiology and Biotechnology, 103, 2329-2338.

Lv, R., Zou, M., Chen, W., Zhou, J., Ding, T., Ye, X., et al. (2019). Ultrasound: Enhance the detachment of exosporium and decrease the hydrophobicity of Bacillus cereus spores. Lebensmittel-Wissenschaft und -Technologie- Food Science and Technology, 116, 108473.

Malyshev, D., \& Baillie, L. (2020). Surface morphology differences in Clostridium difficile spores, based on different strains and methods of purification. Anaerobe, 61, 102078.

Milani, E. A., \& Silva, F. V. M. (2017). Ultrasound assisted thermal pasteurization of beers with different alcohol levels: Inactivation of Saccharomyces cerevisiae ascospores. Journal of Food Engineering, 198, 45-53.

Montville, T. J., \& Sapers, G. M. (1981). Thermal resistance of spores from pH elevating strains of Bacillus licheniformis. Journal of Food Science, 46(6), 1710-1712.

Nakayama, A., Yano, Y., Kobayahi, S., Ishikawa, M., \& Sakai, K. (1996). Comparison of pressure resistance of spores of six Bacillus strains with their heat resistances. Applied and Environmental Microbiology, 62(10), 3897-3900.

National Advisory Committee on Microbial Criteria for Foods. (2006). Requisite scientific parameters for establishing the equivalence of alternative methods of pasteurization. Journal of Food Protection, 69(5), 1190-1216.

Oomes, S. J., van Zuijlen, A. C., Hehenkamp, J. O., Witsenboer, H., van der Vossen, J. M. B. M., \& Brul, S. (2007). The characterisation of Bacillus spores occurring in the manufacturing of (low acid) canned products. International Journal of Food Microbiology, 120(1-2), 85-94.

Ordonez, J., \& Burgos, J. (1976). Effect of ultrasonic waves on the heat resistance of Bacillus spores. Applied and Environmental Microbiology, 32, 183-184.

Owusu-Ansah, P., Yu, X., Osae, R., Zhou, C., Zhang, R., Mustapha, A. T., et al. (2020). Optimization of thermosonication on Bacillus cereus from pork: Effects on inactivation and physicochemical properties. Journal of Food Process Engineering, 43 (6), Article e13401.

Palacios, P., Burgos, J., Hoz, L., Sanz, B., \& Ordóñez, J. A. (1991). Study of substances released by ultrasonic treatment from Bacillus stearothermophilus spores. Journal of Applied Bacteriology, 71, 445-451.

Palanisamy, N., Seale, B., Turner, A., \& Hemar, Y. (2019). Low frequency ultrasound inactivation of thermophilic bacilli (Geobacillus spp. and Anoxybacillusflavithermus) in the presence of sodium hydroxide and hydrogen peroxide. Ultrasonics Sonochemistry, 51, 325-331.

Pavic, S., Brett, M., Petric, N., Lastre, D., Smoljanovic, M., Atkinson, M., et al. (2005). An outbreak of food poisoning in a kindergarten caused by milk powder containing toxigenic Bacillus subtilis and Bacillus licheniformis. Archiv für Lebensmittelhygiene, 56 (1), 20-22.

Peng, Y., \& Lu, R. (2006). An LCTF-based multispectral imaging system for estimation of apple fruit firmness: Part II. Selection of optimal wavelengths and development of prediction models. Transactions of the ASAE, 49, 269-275. 
Pettipher, G. L., \& Osmundson, M. E. (2000). Methods for the detection, enumeration and identification of Alicyclobacillus acidoterrestris. Food Australia, 52(7), 293.

Pinhatti, M. E. M. C., Variane, S., Eguchi, S. Y., \& Manilla, G. E. (1997). Detection of acidothermophilic Bacilli in industrialized fruit juices. Fruit Processing, 7(9), 350-353.

Pitt, J. I., \& Hocking, A. D. (2009). Fungi and food spoilage (3rd ed.). New York: Springer Science \& Business Media.

Piyasena, P., Mohareb, E., \& McKellar, R. (2004). Inactivation of microbes using ultrasound: A review. International Journal of Food Microbiology, 87(3), 207-216.

Rao, L. J. M., \& Ramalakshmi, K. (2011). Quality control. In L. J. M. Rao, \& K. Ramalakshmi (Eds.), Recent trends in soft beverages (pp. 223-235). India: Woodhead Publishing India.

Riesz, P., \& Kondo, T. (1992). Free radical formation induced by ultrasound and its biological implications. Free Radical Biology and Medicine, 13(3), 247-270.

Rodriguez, J. H., Cousin, M. A., \& Nelson, P. E. (1993). Thermal resistance and growth of Bacillus licheniformis and Bacillus subtilis in tomato juice. Journal of Food Protection, 56(2), 165-168.

Rupnik, M., \& Songer, J. G. (2010). Clostridium difficile: Its potential as a source of foodborne disease. Advances in Food \& Nutrition Research, 60, 53-66.

Sagong, H., Cheon, H., Kim, S., Lee, S., Park, K., Chung, M., et al. (2013). Combined effects of ultrasound and surfactants to reduce Bacillus cereus spores on lettuce and carrots. International Journal of Food Microbiology, 160, 367-372.

Salkinoja-Salonen, M. S., Vuorio, R., Andersson, M. A., Kämpfer, P., Andersson, M. C., Honkanen-Buzalski, T., et al. (1999). Toxigenic strains of Bacillus licheniformis related to food poisoning. Applied and Environmental Microbiology, 65, 4637-4645.

Salomão, B. C. M. (2018). Pathogens and spoilage microorganisms in fruit juices: An overview. In G. Rajauria, \& B. K. Tiwari (Eds.), Fruit juices (pp. 291-308). Academic Press.

Sandoval, A. J., Barreiro, J. A., \& Mendoza, S. (1992). Thermal resistance of Bacillus coagulans in double concentrated tomato paste. Journal of Food Science, 57(6), 1369-1370.

Scheldeman, P., Herman, L., Foster, S., \& Heyndrickx, M. (2006). Bacillus sporothermodurans and other highly heat-resistant spore formers in milk. Journal of Applied Microbiology, 101(3), 542-555.

Silva, F. V. M., \& Evelyn. (2018). High pressure processing effect on microorganisms in fruit and vegetable products. In M. Houška, \& F. V. M. Silva (Eds.), High pressure processing of fruit and vegetable products (pp. 343-378). Boca Raton, FL: CRC Press.

Silva, F. V. M., \& Evelyn. (2020). Resistant moulds as pasteurization target for cold distributed high pressure and heat assisted high pressure processed fruit products. Journal of Food Engineering, 282, 109998.

Silva, F. V. M., \& Gibbs, P. (2001). Alicyclobacillus acidoterrestris spores in fruit products and design of pasteurisation processes. Trends in Food Science \& Technology, 12(2), 68-74.

Silva, F. V. M., \& Gibbs, P. (2004). Target selection in designing pasteurization processes for shelf-stable high-acid fruit products. Critical Reviews in Food Science and Nutrition, 44(5), 353-360.

Silva, F. V. M., \& Gibbs, P. A. (2009). Principles of thermal processing: Pasteurisation. In R. Simpson (Ed.), Engineering aspects of thermal food processing (pp. 14-38). Boca Raton, FL: CRC Press.
Silva, F. V. M., \& Gibbs, P. (2010). Non-proteolytic Clostridium botulinum spores in lowacid cold-distributed foods and design of pasteurization processes. Trends in Food Science \& Technology, 21(2), 95-105.

Silva, F. V. M., Gibbs, P. A., Nunez, H., Almonacid, S., \& Simpson, R. (2014). Thermal processes: Pasteurization. In C. A. Batt, \& M. L. Tortorello (Eds.), Encyclopedia of food microbiology (pp. 577-595). Amsterdam: Elsevier.

Silva, F. M., Gibbs, P., Vieira, M. C., \& Silva, C. L. M. (1999). Thermal inactivation of Alicyclobacillus acidoterrestris spores under different temperature, soluble solids and $\mathrm{pH}$ conditions for the design of fruit processes. International Journal of Food Microbiology, 51(2/3), 95-103.

Silva, F. V. M., \& Sulaiman, A. (2017). Advances in thermosonication for the inactivation of endogenous enzymes in foods. In D. Bermudez-Aguirre (Ed.), Ultrasound: Advances in food processing and preservation (pp. 101-130). Academic Press.

Sulaiman, A., Soo, M. J., Farid, M., \& Silva, F. V. M. (2015). Thermosonication for polyphenoloxidase inactivation in fruits: Modeling the ultrasound and thermal kinetics in pear, apple and strawberry purees at different temperatures. Journal of Food Engineering, 165, 133-140.

Sussman, A. S., \& Halvorson, H. O. (1966). Spores: Their dormancy and germination. New York: Harper \& Row.

Therre, H. (1999). Botulism in the European union. Euro Surveillance, 4(1), 2.

Tiwari, B. K. (2015). Ultrasound: A clean, green extraction technology. Trends in Analytical Chemistry, 71, 100-109.

Tiwari, B. K., \& Mason, T. J. (2012). Ultrasound processing of fluid foods. In P. J. Cullen, B. K. Tiwari, \& V. P. Valdramidis (Eds.), Novel thermal and non-thermal technologies for fluid foods (pp. 135-165). Academic Press.

Tournas, V. (1994). Heat-resistant fungi of importance to the food and beverage industry, Critical Reviews in Microbiology, 20, 243-263.

Tremarin, A., Brandão, T. R. S., \& Silva, C. L. M. (2017). Application of ultraviolet radiation and ultrasound treatments for Alicyclobacillus acidoterrestris spores inactivation in apple juice. Lebensmittel-Wissenschaft und -Technologie- Food Science and Technology, 78, 138-142.

Tremarin, A., Canbaz, E. A., Brandão, T. R. S., \& Silva, C. L. M. (2019). Modelling Alicyclobacillus acidoterrestris inactivation in apple juice using thermosonication treatments. Lebensmittel-Wissenschaft und -Technologie- Food Science and Technology, 102, 159-163.

Van Boekel, M. A. J. S. (2002). On the use of the Weibull model to describe thermal inactivation of microbial vegetative cells. International Journal of Food Microbiology, 74, 139-159.

Walls, I., \& Chuyate, R. (1998). Alicyclobacillus-historical perspective and preliminary characterization study. Dairy, Food, and Environmental Sanitation, 18, 499-503.

Weingart, O. G., Schreiber, T., Mascher, C., Pauly, D., Dorner, M. B., Berger, T. F. H., et al. (2010). The case of Botulinum toxin in milk: Experimental data. Applied and Environmental Microbiology, 76(10), 3293-3300.

Wordon, B. A., Mortimer, B., \& Mcmaster, L. D. (2012). Comparative real-time analysis of Saccharomyces cerevisiae cell viability, injury and death induced by ultrasound ( 20 $\mathrm{kHz}$ ) and heat for the application of hurdle technology. Food Research International, 47, 134-139.

Wyatt, T. T., Leeuwen, M. R., Golovina, E. A., Hoekstra, F. A., Kuenstner, E. J., Palumbo, E. A., et al. (2015). Functionality and prevalence of trehalose-based oligosaccharides as novel compatible solutes in ascospores of Neosartorya fischeri (Aspergillus fischeri) and other fungi. Environmental Microbiology, 17(2), 395-411. 